\title{
La pobreza en España en el período 1985-1995. Un análisis transversal y longitudinal
}

\author{
$\mathrm{M}^{\mathrm{a}}$. ROSARIO GONZÁLEZ RODRÍGUEZ, FRANCISCO VELASCO MORENTE \\ y LUIS GONZÁLEZ ABRIL \\ Departamento Economía Aplicada I \\ UNIVERSIDAD DE SEVILLA \\ e-mail: rosaglez@us.es; velasco@us.es y luisgon@us.es
}

\begin{abstract}
RESUMEN
El objetivo del trabajo consiste en un análisis transversal y longitudinal de la situación de pobreza económica experimentada por los hogares españoles en el período 1985-1995, utilizando para ello la Encuesta Continua de Presupuestos Familiares en dicho período. En el enfoque transversal se han analizado los tres aspectos de la pobreza: el aspecto absoluto, el relativo y la desigualdad en la cola inferior de la distribución. El análisis se ha llevado a cabo mediante la construcción de índices de pobreza y la representación gráfica de las curvas TIPs. El análisis longitudinal se ha basado en la estimación de las tasas de salida de la pobreza y la función de supervivencia, permitiendo conocer la duración en el estado de pobreza, las transiciones al estado de "no pobreza" e identificar aquellas características socioeconómicas y demográficas del hogar con mayor influencia en la duración de la pobreza.
\end{abstract}

Palabras clave: Métodos paramétricos; curvas TIP's; estimación Kaplan-Meier.

\section{Poverty in Spain in the Period 1985-1995. A Cross-Sectional and Longitudinal Analysis}

\begin{abstract}
The objective of the article has consisted of a cross-sectional and longitudinal analysis of the economic poverty state during the period 1985-1995, using the Panel Household Budget Survey for this period of time. The three aspects of poverty incidence, intensity and inequality in the left tail of the distribution, have been analysed in the cross-sectional approach. This analysis has been carried out through the calculations of poverty indices and the graphical representation of the TIPs curves. The longitudinal analysis, has focussed on the estimation of the risk function and the survival function, which allows us to know the duration in poverty, the transitions out of poverty and to identify those socio-economic and demographic characteristics of household with more influence in the duration of poverty.

Keywords: Non Parametric Methods; TIP's Curves; Kaplan-Meier Estimation.
\end{abstract}

Clasificación JEL: C14, C41, D19, I32.

Artículo recibido en enero de 2006 y aceptado para su publicación en febrero de 2008.

Artículo disponible en versión electrónica en la página www.revista-eea.net, ref. อ-26211. 


\section{INTRODUCCIÓN}

Desde los años 70 se ha venido observando en la literatura una gran cantidad de trabajos empíricos que permiten cuantificar la pobreza y la desigualdad, así como identificar las características socioeconómicas de la población pobre en un momento específico del tiempo en una zona geográfica, utilizándose para ello bases de datos de corte transversal como las Encuestas de Presupuestos Familiares básicamente anuales [Deleeck y de Bosch (1990), Escribano (1990), Mercader (1995), INE (1996)]. Recientemente la disponibilidad de datos de Panel hace más asequible el análisis del fenómeno de la pobreza y la desigualdad de forma más precisa y detallada. En este sentido, dichas encuestas permiten determinar si la pobreza en una determinada región es una situación a corto plazo (pobreza transitoria), debido a factores negativos como es el caso de depresión en el ciclo económico, o si por el contrario es una situación a largo plazo (pobreza permanente), es decir, si constituye una condición estructural que se transmite de generación en generación.

La distinción entre pobreza transitoria y pobreza permanente es de vital impartancia en la actualidad, sobre todo en los países industrializados en los que un gran número de hogares experimentan períodos cortos de pobreza a lo largo de sus vidas [Stevens (1999), Cantó y otros (2003), Barcena y Cowell (2006)]. Además, esta distinción es crucial ya que si bien la pobreza transitoria aparece como un fenómeno que tiende a solventarse por sí mismo, la pobreza permanente constituye un problema mucho más grave que requiere la acción de políticas públicas específicas para la reducción de la misma.

El objetivo perseguido en este trabajo se centra en abordar un análisis estático y dinámico de la pobreza en España en el período comprendido desde el primer trimestre del año 1985 hasta el cuarto trimestre del año 1995. En el análisis estático se han obtenido diferentes índices de pobreza y se ha utilizado la metodología de las curvas TIPs a fin de analizar la trayectoria de la pobreza económica en el período de estudio. En el análisis dinámico se ha utilizado un enfoque no paramétrico basado en las estimaciones de la función riesgo y de la función de supervivencia permitiendo analizar la movilidad en la parte baja de la distribución de la renta, así como identificar aquellas características socioeconómicas de los hogares que más incidían en dicha movilidad.

El período de estudio abarcado en este trabajo comprende desde el año 1985 hasta el 1995, comienzo y final de la primera etapa socialista en el gobierno de España tras la firma de la Constitución del año 1978. Señalar que en el año 1985 el gobierno socialista que llevaba dos años y tres meses de gobierno abordó medidas sociales en relación al problema de la pobreza. El desempleo, problemática estrechamente unida a la pobreza aumentó en este período empezando a bajar en 1990. En el año 1996 finaliza un primer ciclo de gobierno socialista con la entrada al poder de un gobierno de centro-derecha.

El trabajo se ha estructurado como sigue. En la sección segunda se describen los aspectos metodológicos utilizados en la medición de la pobreza económica. En 
la sección tercera se aborda el análisis estático exponiéndose la metodología utilizada, así como los principales resultados obtenidos. El análisis dinámico se realiza en la sección cuarta, obteniéndose estimaciones de las tasas de salida de pobreza y de la función de supervivencia, e identificando aquellas características socioeconómicas y demográficas del hogar con mayor incidencia en la duración en pobreza. Por último, se recogen las principales conclusiones del trabajo.

\section{ASPECTOS METODOLÓGICOS}

\subsection{Base de datos}

La Encuesta Continua de Presupuestos Familiares (ECPF) [INE (1985)] es una encuesta de panel trimestral con carácter rotatorio, iniciada por el Instituto de Nacional de Estadística (INE) de España en enero de 1985. La unidad de observación es el hogar, entendiendo como tal la persona o conjunto de personas que ocupan en común una vivienda familiar principal o parte de ella, consumen, comparten alimentos y otros bienes con cargo a un mismo presupuesto. Su principal objetivo es proporcionar información sobre los presupuestos de los hogares, con el fin de adaptar las ponderaciones de los bienes de consumo para el cálculo del índice de Precios al Consumo (IPC). Además, ofrece información sobre los ingresos de carácter monetario y no monetario de los hogares, permitiendo el análisis de la desigualdad y la pobreza en términos de ingresos o gastos, así como la obtención de elasticidades gasto-renta de los mismos. También se hallan recogidas en dichas encuestas, datos relativos a las características demográficas de la unidad de análisis (número de miembros del hogar, número de perceptores de ingresos, tamaño del municipio en el que habitan, etc.), e información sobre características socioeconómicas del sustentador principal (edad, categoría laboral del sustentador principal, nivel de estudios del sustentador, etc.).

El ámbito geográfico de la encuesta es todo el territorio nacional español, por lo que se estudian hogares en todas las Comunidades Autónomas. El ámbito temporal sometido a estudio es cada ciclo trimestral del año; los datos se recopilan en cada uno de los trimestres del año y los hogares que se mantienen en el panel son encuestados un máximo de dos años (ocho trimestres consecutivos). La sustitución de los hogares se realiza mediante un proceso de rotación, renovándose éstos parcialmente cada trimestre (una octava parte de la muestra cada trimestre), a fin de evitar el cansancio o perversión de los miembros de los hogares encuestados.

En principio, la ECPF contiene los datos necesarios para realizar un análisis de los fenómenos de pobreza y desigualdad, conocer la duración en el estado de pobreza, identificar los factores que inciden en esas duraciones, así como su posible relación con la evolución de los ciclos económicos, particularmente de período corto. No obstante, hay que destacar algunas limitaciones importantes de la ECPF 
en los estudios de pobreza de carácter longitudinal. La primera se debe al hecho de que los hogares son encuestados como máximo dos años, ${ }^{1}$ período corto para la identificación de la pobreza permanente propiamente dicha. ${ }^{2}$ Por otra parte, la ECPF, al igual que ocurría con las EPFs (Encuesta de Presupuestos Familiares) excluye en el diseño muestral los estratos más marginados de la población, lo que condiciona de alguna manera los resultados obtenidos al subestimar la pobreza más severa [Ruiz-Huerta y Martínez (1994), Martín Guzmán y otros (1996)].

\subsection{Unidad de Análisis}

La unidad técnica de análisis utilizada en los estudios de pobreza de corte transversal es el "hogar" o la "familia" [Citro y Michel (1995)], entre otras razones, porque el bienestar de los miembros dependen fuertemente del bienestar del hogar, ya que dicha unidad actúa como filtro en el proceso de transformación de cualquier fuente de ingreso en bienestar. Sin embargo, la unidad de observación en estudios de pobreza de carácter longitudinal es el "individuo", al admitirse que los hogares al cambiar frecuentemente su composición no constituyen una unidad de observación estable en el tiempo. En el estudio longitudinal de la pobreza de este trabajo se ha utilizado, no obstante, el hogar como unidad de análisis dado que en las ECPFs sólo se dispone de información de la trayectoria de los hogares en el tiempo ${ }^{3}$ y no de los individuos [INE (1985)], a diferencia de otras Encuestas de Panel para las que si existe dicha información [Panel Survey of Income Dynamics (PSID) de Estados Unidos, British Household Panel Survey (BHPS) de Reino Unido, German Socioeconomic Panel (GSOEP) de Alemania, Panel de hogares de la Unión Europea (PHOGUE)].

Por otro lado, en ausencia de información precisa de cuál es el mecanismo mediante el cual los ingresos del hogar se comparten entre los distintos miembros del hogar, se asume que los ingresos son compartidos por igual entre todos los miembros del hogar, por lo que a cada miembro del hogar se le asigna no su ingreso personal sino una cantidad de dinero por adulto equivalente.

\footnotetext{
${ }^{1}$ Como consecuencia de la estructura trimestral de la encuesta, se observa una importante tasa de abandono de los hogares encuestados (attrition), lo que implica la pérdida de información en el análisis longitudinal de la pobreza. No obstante, hay que destacar que la tasa de abandono no está correlacionada con el nivel de ingresos del hogar [Cantó (2003)].

${ }^{2}$ El Panel de Hogares de la Unión Europea (PHOGUE) tiene una cobertura anual corrigiendo esta limitación de la ECPF. No obstante, para el caso de España se dispone de información desde 1993, no cubriendo el período 1985-1995 para el que deseamos analizar la evolución estática y dinámica de la pobreza. Sobre este tema, se puede consultar Bárcena y Cowell (2006) donde se realiza un análisis estático y dinámico de la pobreza en España para el período 1993-2000 utilizándose el PHOGUE español.

${ }^{3}$ Dado que en las ECPFs el hogar se sigue como máximo dos años, se ha observado que la composición del mismo es prácticamente estable en dicho período.
} 


\subsection{Línea de Pobreza}

Para definir el umbral de pobreza elegimos el ingreso disponible del hogar como indicador del bienestar económico del mismo al medir las oportunidades del hogar $\mathrm{y}$, por tanto, la de sus miembros a acceder a una vida digna [Sen (1992]. De esta forma, se define la variable ingreso total disponible del hogar ${ }^{4}$ como la suma de todas las fuentes de ingreso de sus miembros.

Dado que los hogares difieren en composición y tamaño, se define el "ingreso del hogar por adulto equivalente" para el trimestre $T, y_{h T}$, de acuerdo con la siguiente expresión:

$$
y_{h T}=\frac{x_{h T}}{n_{h T}^{s}}
$$

donde $x_{h T}$ representa el ingreso no ajustado del hogar $h$ en el trimestre $T, n_{h T}$ el número de miembros del hogar h en el trimestre $T$ y s un parámetro que varía entre 0 y 1 , que se interpreta como la elasticidad de las necesidades del hogar respecto de su tamaño. Para la estimación del parámetro $s$ se ha ajustado el modelo, sin término independiente [Buhmann et al. (1988)]:

$$
\ln \left(E_{h}\right)=s \ln \left(n_{h}\right)+e_{h} \quad h=1, \cdots, N
$$

donde $N$ es el total de hogares en la muestra objeto de estudio. La variable $E_{h}$ es el número de adultos equivalentes calculado para cada hogar utilizando la escala de la $\mathrm{OCDE}^{5}$ y $e_{h}$ representa el término de error. La estimación del parámetro para el caso de España utilizando la ECPF es de 0,78.

Por otro lado y puesto que no existe un acuerdo generalizado sobre qué línea de pobreza se ha de utilizar en estudios de carácter longitudinal [Heinrich (1999)], en el estudio hemos optado por utilizar aquella línea de pobreza que combina el aspecto absoluto y relativo de la pobreza [Cantó (1996)]:

$$
p l_{i}=p l_{1} \prod_{j}\left(1-\theta_{j}\right) \quad i=2, \cdots, 8
$$

donde, $p l_{i}$ es la línea de pobreza para el trimestre i-ésimo, $p l_{1}$ es la línea de pobreza para el primer trimestre de 1985 , definida como el $50 \%$ de la mediana del ingreso por adulto equivalente en ese trimestre, y $\theta_{j}$ es la tasa de inflación trimestral.

\footnotetext{
${ }^{4}$ De acuerdo con la ECPFs, los ingresos monetarios que el hogar debe considerar como percibidos, serán los que resulten una vez deducidas las cantidades satisfechas en concepto de impuestos y otros pagos asimilados (Seguridad Social, mutualidades, etc.).

${ }^{5}$ La Escala de la OCDE asigna el valor 1 al sustentador principal, 0,7 a los restantes miembros adultos del hogar y 0,5 para los niños (de edad inferior a 14 años). Así, el número de adultos equivalente del hogar $h$, es calculado utilizando la expresión,
}

$$
E_{h}=\left[1+\left(N_{a d}-1\right) \times 0,7+N_{c h} \times 0,5\right]
$$

siendo $N_{a d}$, número de adultos excluido el sustentador principal y $N_{c h}$ el número de niños del hogar. 
La línea de pobreza así definida combina el carácter relativo al tomar la mediana del ingreso por adulto equivalente del primer trimestre de 1985, y el carácter absoluto porque la mediana del trimestre considerado se mantiene para los siguientes trimestres. Además su elección se justifica porque dicha línea no impone un nivel exógeno del ingreso por adulto equivalente "necesario" y porque evita el efecto del crecimiento de la mediana en el tiempo. ${ }^{6}$

\section{ANÁLISIS ESTÁTICO DE LA POBREZA}

A fin de analizar la trayectoria de la pobreza económica experimentada por los hogares españoles en el período que comprende el primer trimestre de 1985 hasta el cuarto trimestre de 1995, empleamos distintos índices de pobreza con objeto de obtener conclusiones más robustas.

Índice de Incidencia de la Pobreza, -Head count ratio- (H),

$$
H=\frac{q}{N}
$$

donde $q$ es el número de pobres y $N$ el total de la población.

A pesar de ser muy utilizado en la medición de la pobreza, ${ }^{7}$ presenta impartantes limitaciones [Sen (1976)], dado que sólo considera un aspecto de la pobreza, la extensión de la misma (aspecto absoluto de la pobreza), siendo insensible al grado o intensidad de pobreza de los pobres. Por otra parte, no satisface el Axioma de Transferencias, y como se deduce de su expresión analítica, no recoge la desigualdad de la renta entre los pobres y, por tanto, es insensible a cualquier cambio en la distribución del ingreso.

Índice de Insuficiencia de Renta, - Poverty gap ratio- (I),

$$
I=\frac{1}{q} \sum_{i=1}^{q} \frac{z-y_{i}}{z}
$$

siendo $z$ la línea (o umbral) de pobreza, $y_{i}$ el ingreso del hogar $i$-ésimo y $q$ el número pobres.

Este índice, que cuantifica la intensidad de la pobreza (aspecto relativo de la pobreza), presenta también importantes limitaciones [Sen (1976)] dado que no recoge el aspecto absoluto de pobreza, no satisface el Axioma de Transferencias $\mathrm{y}$ es insensible a la desigualdad de renta entre los pobres. No obstante, y al igual que ocurría con el índice de incidencia de la pobreza, $H$, esta medida es muy utilizada en los estudios empíricos y se presenta acompañando a otras medidas de pobreza debido a su sencillez de cálculo e interpretación.

\footnotetext{
${ }^{6}$ La elección de la mediana en lugar de la media del ingreso por adulto equivalente es debido a que la mediana es más robusta que la media ante valores anómalos de la variable.

${ }^{7}$ Actualmente se sigue utilizando incluso para comparaciones inter-temporales e internacionales y suele acompañar a las medidas globales de pobreza en todo estudio empírico.
} 
Con el fin de corregir las deficiencias que presentan los anteriores índices de medición de la pobreza, surgen en la literatura índices agregados que tratan de recoger los tres aspectos básicos de la pobreza: el absoluto (proporción de pobres), el aspecto relativo (la intensidad de la pobreza), y la desigualdad de la distribución de los ingresos entre los pobres; y que al mismo tiempo verifican propiedades deseables para un buen índice de pobreza. En este trabajo hemos optado por la familia de índices de Foster, Greer and Thobercke (1984).

Índice de Foster, Greer y Thobercke (1984)-(FGT),

$$
F G T_{\alpha}=\frac{1}{N} \sum_{i=1}^{q}\left(\frac{z-y_{i}}{z}\right)^{\alpha-1} \quad \alpha>0
$$

Esta familia de índices ponderan la importancia relativa del desnivel de pobreza respecto del umbral $z$, ya que a medida que $\alpha$ aumenta crece la importancia relativa que el índice concede a los desniveles de pobreza más fuertes, por lo que $\alpha$ se interpreta como el parámetro de aversión a la pobreza. Se puede observar que $H=F G T_{1}$ y $H I=F G T_{2}$. El índice tendría la siguiente expresión para $\alpha=3$ :

$$
F G T_{3}=H\left[I^{2}+(1-I) C V_{p}^{2}\right]
$$

donde $C V_{p}$ es el coeficiente de variación entre las unidades pobres. El índice de Foster-Greer-Thobercke satisface el Axioma de sensibilidad decreciente a las transferencias para un valor del parámetro mayor a tres $(\alpha>3)$, por lo que el índice $\mathrm{FGT}_{4}$ es muy utilizado cuando se pretende prestar atención a los más pobres.

Con el fin de ilustrar la trayectoria de la pobreza en España emplearemos también la metodología expuesta en Jenkins y Lambert (1997), comparando lo acaecido en la etapa de crecimiento económico con la etapa de recesión en el período 1985-1995. Esta metodología permite comparaciones inter-temporales en una misma área geográfica y, por consiguiente, la posibilidad de ordenación en términos de pobreza entre distintos momentos del tiempo. ${ }^{8}$ El enfoque de Jenkins y Lambert consiste en definir las denominadas curvas TIP's -the Three I of Poverty curves- como aquellas curvas que están basadas en la distribución de los gaps de pobreza proporcionando una representación gráfica de los tres aspectos de la pobreza: incidencia, intensidad y desigualdad. Estas curvas permiten al mismo tiempo, una ordenación en la pobreza ${ }^{9}$ de acuerdo con una clase particular de índices [Del Rio y Ruiz-Castillo (2001)].

En la Figura 1 se muestra una curva TIP teórica. La incidencia de la pobreza está representada por la longitud de la curva TIP, la intensidad viene representada por la altura de la curva, y el aspecto de la desigualdad está resumido por el grado de concavidad de la parte no horizontal de la curva.

\footnotetext{
${ }^{8}$ La metodología propuesta no sólo permite comparaciones inter-temporales sino también interregionales e inter-grupos (Jenkins y Lambert 1998).

${ }^{9}$ Jenkins y Lambert demuestran que las ordenaciones son robustas ante variaciones en el deflactor utilizado.
} 
FIGURA 1

Representación gráfica de una Curva TIP.

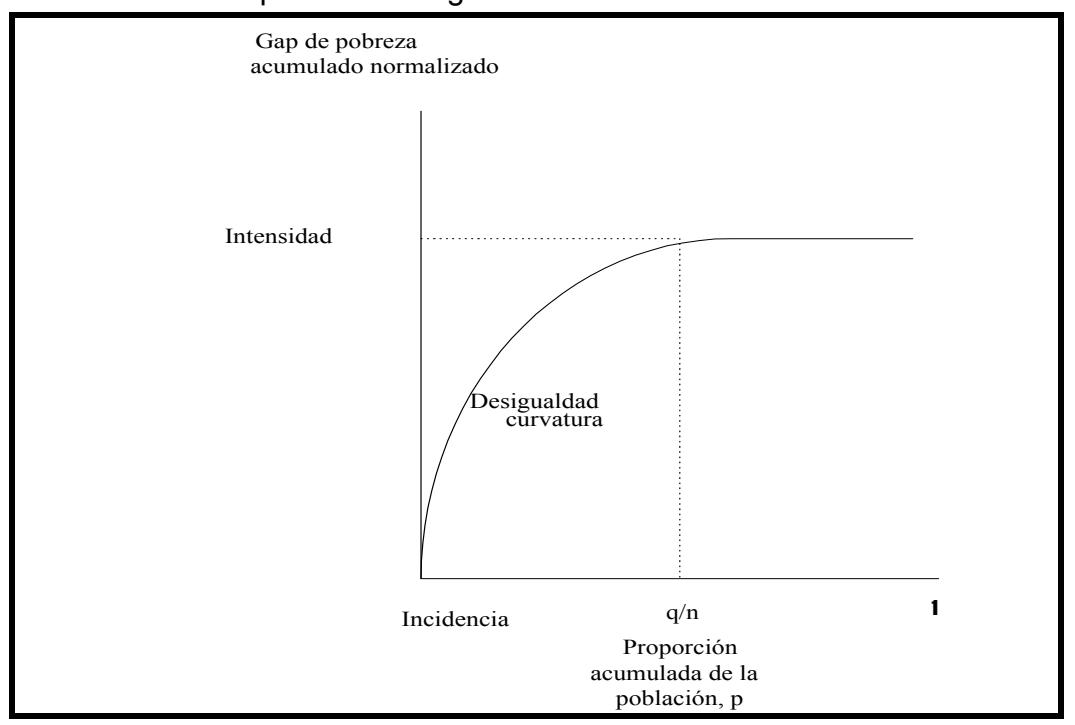

\subsection{Análisis Estadístico. Principales resultados del Análisis Estático}

En la Tabla 1 se recoge los valores de los índices de pobreza de incidencia, intensidad y el índice de $F G T_{3}$ en el período de estudio. Se observa que en los cuatro trimestres de 1985 nos encontramos con las mayores proporciones de hogares pobres, y además estos sufren pobreza con mayor intensidad que en el resto de los años. En el período comprendido entre el segundo trimestre de 1989 y el segundo trimestre de 1993 se registra la menor tasa de pobreza, aproximadamente 8\% de los hogares se encuentran por debajo del umbral de pobreza. Además, en este período el colectivo de pobres experimenta pobreza económica con menor intensidad que en el resto del período, como puede observarse de la trayectoria seguida por el índice de Intensidad de la pobreza (I) y el índice de Foster-Greer-Thobercke $\left(F G T_{3}\right)$. A partir del segundo trimestre de 1993, la proporción de pobres crece suavemente hasta alcanzar aproximadamente el $9 \%$ en el cuarto trimestre de 1995. Se observa también esta trayectoria en los índices de intensidad y de Foster-Greer-Thobercke. 


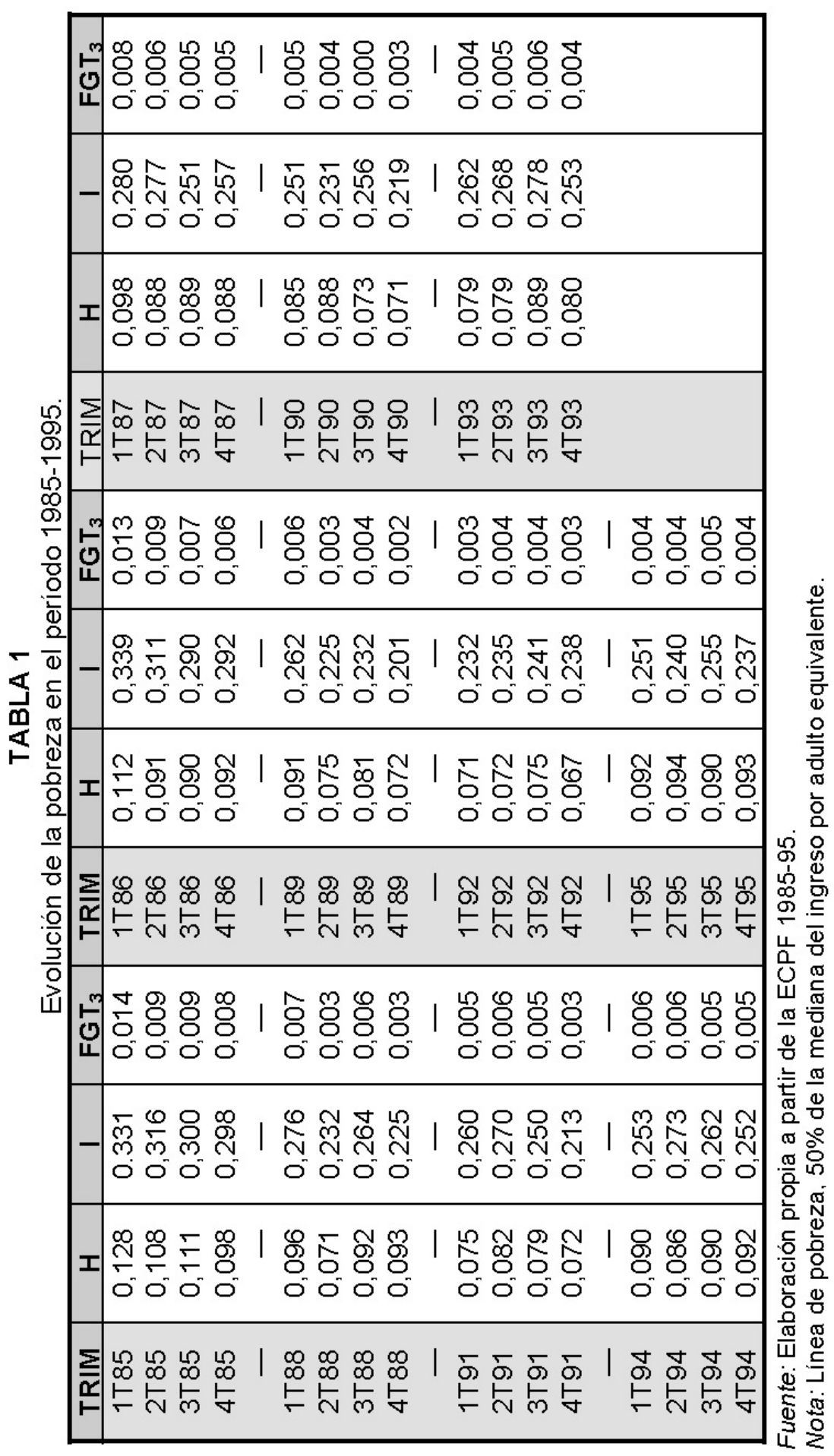


Para ilustrar la dominancia en pobreza hemos elegido algunos trimestres correspondientes al período de crecimiento económico, 1986-1989, y otros correspondientes al período de recesión, segundo semestre 1992-1993.

FIGURA 2

Curvas TIPs para el 4 ${ }^{\circ}$ Trimestre 1987-4º trimestre 1992.

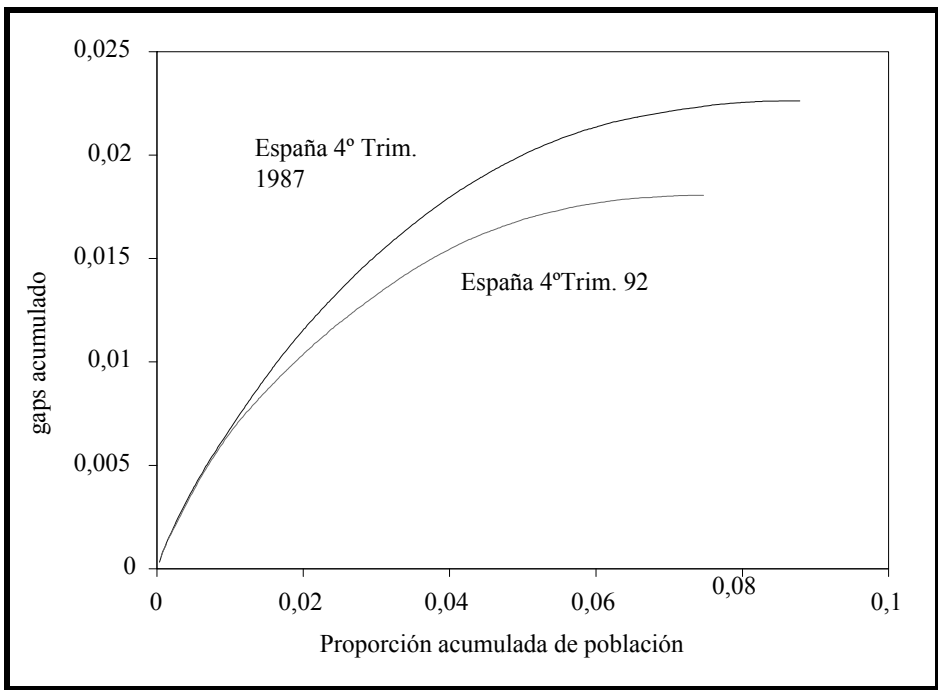

FIGURA 3

Curvas TIPs para el $4^{\circ}$ trimestre $1988-4^{\circ}$ trimestre 1992.

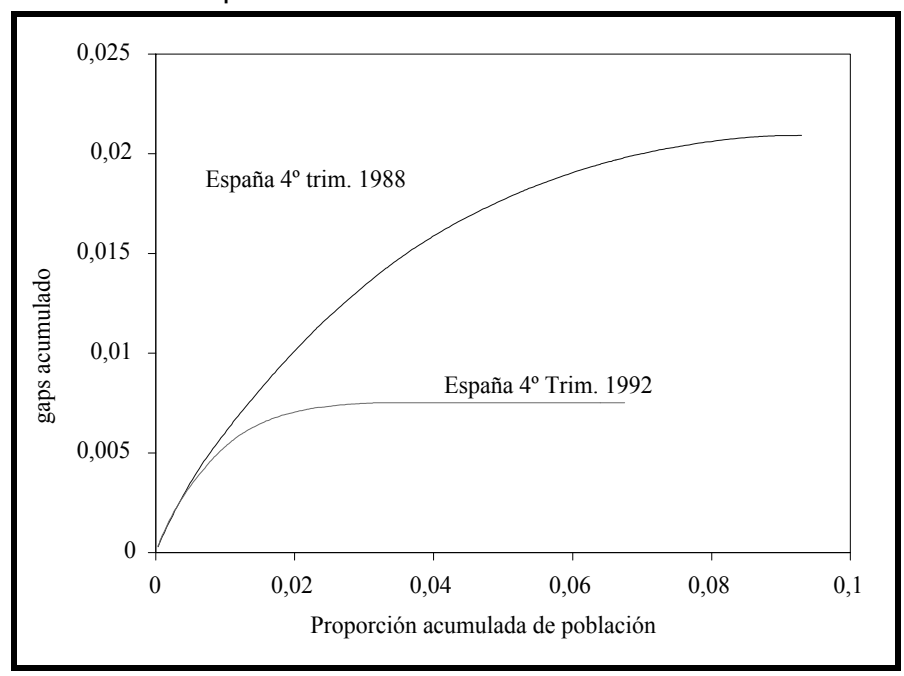


En las Figuras 2 y 3 se observa cómo la distribución de las intensidades de pobreza en el período de crecimiento domina a la distribución de las intensidades de pobreza en el período de recesión, es decir, la etapa expansiva de la economía no fue acompañada por una reducción de los pobres y menos aún de una disminución en la intensidad de la pobreza. ${ }^{10}$

\section{ANÁLISIS DINÁMICO DE LA POBREZA}

El Análisis de Supervivencia, el cual debe su denominación a que han sido frecuentemente utilizados para el estudio de fallecimientos, constituye una clase de métodos estadísticos para el estudio de la ocurrencia y duración de los sucesos. Este tipo de análisis es extremadamente útil para el estudio de diferentes sucesos, tanto en ciencias sociales como en ciencias naturales, incluyendo por ejemplo, el comienzo de una enfermedad, fallos del equipamiento, divorcios, jubilación, salida del mercado de trabajo, crash del mercado de acciones, duración de las huelgas, etc. Estos métodos estadísticos reciben distintas denominaciones al ser adaptados $\mathrm{y}$ a veces redescubiertos independientemente por los investigadores en distintos campos. Así, recibe el nombre de "Análisis de la historia de los sucesos" (Event History Analysis) en Sociología [Hosmer y Lemeshow (1999)], "Análisis de Fiabilidad" (Reliability Analysis) y "Análisis de Tiempo de Fallo" en Ingeniería [Kalbfleische y Prentice (1980)], "Análisis de Duración" (Duration Analysis) y Análisis de Transición" (Transition Analysis) en Economía [Heckaman y Singer (1984); Kiefer (1988)]. La aplicación estadística de estos métodos en ciencias sociales comenzó hacia finales de los años setenta y durante los últimos 15 años han proliferado trabajos en los que se analizan cuestiones tales como el tiempo que un individuo permanece en desempleo [Ahn y Ugidos (1995)], duración de una patente [Pakes (1989)], duración de huelgas [Kennan (1985)], duración en pobreza [Duncan (1993), Jenkins et al. (2001), Cantó (2003)], etc.

Con los modelos de duración se intenta analizar las transiciones que experimenta la unidad de análisis entre dos estados, inicial y final. La variable de interés en el Análisis de Duración es precisamente la variable "duración", es decir, el tiempo de permanencia en un estado inicial y que finaliza cuando la unidad de referencia pasa al estado final. En nuestro análisis, el estado inicial es la situación de pobreza económica, y el estado final se corresponde con la salida de la pobreza. A la "duración en pobreza" o "tiempo de permanencia en pobreza" de un hogar, que es una variable aleatoria, la denotaremos por $T$. Esta es una variable absolutamente continua con valores en el intervalo $[0,+\infty)$, tomando el valor cero en el momento en que el hogar entra en el estado de pobreza, y $P(T=\infty)=0$.

\footnotetext{
${ }^{10}$ Además de las proporcionadas en las anteriores figuras hemos realizados otras comparativas eligiendo un trimestre del período de aceleración económico con otro de desacelaración, lo cual nos ha permitido concluir que la distribución de los gaps de pobreza en un trimestre del período expansivo domina a la distribución de los gaps de pobreza del período de recesión.
} 
Nuestro análisis abarca el período 1985-1995, como consecuencia de ello aparece una característica común a los datos de duración, la "censura". Posibles esquemas de censura son "censura a la derecha" y "censura a la izquierda". La censura a la derecha tiene lugar en el caso en que nos ocupa, cuando el hogar todavía permanece en pobreza cuando el período de estudio ha terminado. La censura a la izquierda aparece cuando los hogares ya se encontraban en el estado de pobreza en el momento en que se comenzó el estudio.

En el contexto de los modelos de duración adquieren relevancia además de la función de densidad, $f(t)$, y la función de distribución, $F(t)$, la función de supervivencia o permanencia, y la función riesgo o de salida de la variable $T$, [Lancaster (1990), Lawless (1982)].

Siendo la función de distribución, $F(t)=P(T \leq t)$, la función de supervivencia o de permanencia de la variable $T$, denotada por $S$, se define como:

$$
S(t)=P(T>t)=1-F(t) .
$$

Esta función nos proporciona la probabilidad de que la duración en el estado de pobreza sea mayor que un tiempo $t$. Se caracteriza porque es una función no creciente, que toma el valor uno en $T=0$ y el valor cero en $T=\infty$.

La función de densidad $f(t)$, viene dada por:

$$
f(t)=\lim _{\Delta t \rightarrow 0} \frac{P(t \leq T \leq t+\Delta t)}{\Delta t}=\frac{\partial F(t)}{\partial t}=-\frac{\partial S(t)}{\partial t}
$$

donde $f(t) \Delta t$ representa la probabilidad de salir del estado de pobreza en el intervalo $[t, t+\Delta t]$.

La función riesgo o de salida de la variable $T$, denotada por $h$, se define como:

$$
h(t)=\lim _{\Delta t \rightarrow 0} \frac{P(t \leq T \leq t+\Delta t / T \geq t)}{\Delta t}
$$

Y, por tanto, $h \cdot \Delta t$ se puede interpretar como la probabilidad de que un hogar en pobreza en el momento $t$ salga de la pobreza en el instante inmediatamente posterior.

Por otro lado, si denominamos $P(A)$ a la probabilidad de salir del estado de pobreza en el intervalo $[t, t+\Delta t], f(t) \Delta t$, y $P(B)$ a la probabilidad de permanecer en el estado hasta $t$, entonces la probabilidad de salir en el intervalo $[t, t+\Delta t]$ sabiendo que ha permanecido hasta $t, S(t)$, se puede obtener a partir de la definición de la probabilidad condicionada:

$$
P(A / B)=P(A \cap B) / P(B)=P(B / A) / P(B)=P(A) / P(B)
$$

ya que $P(B / A)=1$. Dado que $P(A / B)=h(t) \Delta t$, se sigue que:

$$
h(t)=\frac{f(t)}{S(t)} .
$$


Por último, creemos conveniente resaltar la siguiente relación entre la función riesgo y la función de supervivencia,

$$
h(t)=\frac{f(t)}{1-F(t)}=\frac{-\partial[1-F(t)] / \partial t}{1-F(t)}=\frac{\partial\{-\ln [1-F(t)]\}}{\partial t}=\frac{\partial\{-\ln [S(t)]\}}{\partial t}
$$

En la literatura se pueden encontrar trabajos que estudian la duración en el estado de pobreza aplicando métodos no paramétricos [Bane y Ellwood (1983)] y métodos paramétricos [Ruggles y Williams (1989), Stevens (1994)]. En este trabajo hemos optado por un análisis no paramétrico dado que en nuestro estudio no necesitamos asumir una forma funcional específica para la función riesgo.

\subsection{Análisis Estadístico de las duraciones}

En el análisis empírico de la dinámica de la pobreza ha sido necesario, en primer lugar, el cálculo de los períodos de pobreza, para después obtener las estimaciones de la función riesgo y la función de supervivencia. Utilizando la línea de pobreza previamente definida se han clasificado a los hogares en aquellos que experimentan pobreza económica y los que no se hallan en tal situación. Como consecuencia de la rotación de los hogares en la muestra en el período contemplado, fue preciso enlazar los hogares durante 8 trimestres consecutivos, al ser éste el máximo período que los hogares permanecen en la muestra, para observar sus cambios de estado pobres/no pobres, y así poder calcular los períodos de pobreza. Para una muestra de 26.782 hogares, se ha observado que 743 hogares fueron pobres los 8 trimestres consecutivos ${ }^{11}$ y que 4750 hogares salen de la pobreza algún trimestre. En el análisis dinámico hemos tenido sólo en consideración aquellos hogares que sufrieron al menos una transición al estado de no pobreza.

Con la sub-muestra de los hogares que estuvieron al menos un trimestre en pobreza (4750 hogares) hemos calculado la duración de los períodos de pobreza e identificado el tipo de censura, obteniéndose la distribución de períodos de pobreza y censura. ${ }^{12}$

TABLA 2

Distribución de los períodos de pobreza y censura (Total 5695 períodos).

\begin{tabular}{|c|c|c|c|}
\hline No Censurados & $\begin{array}{c}\text { Censurados } \\
\text { Izquierda }\end{array}$ & $\begin{array}{c}\text { Censurados } \\
\text { Derecha }\end{array}$ & $\begin{array}{c}\text { Censurados } \\
\text { Izquierda-Derecha }\end{array}$ \\
\hline 2675 & 207 & 1106 & 1707 \\
\hline
\end{tabular}

Fuente: Elaboración propia a partir de la ECPF 1985-95.

\footnotetext{
${ }^{11}$ Se sigue que estas observaciones se encuentran censuradas a izquierda y derecha.

${ }^{12}$ Algunos hogares se caracterizaron por experimentar varios períodos de pobreza (constituyendo períodos de pobreza múltiples), es decir, durante los ocho trimestres entraron y escaparon de la pobreza varias veces. El tratamiento de los hogares con más de un período ha consistido en incorporarlos a la muestra una vez por cada período de observación.
} 


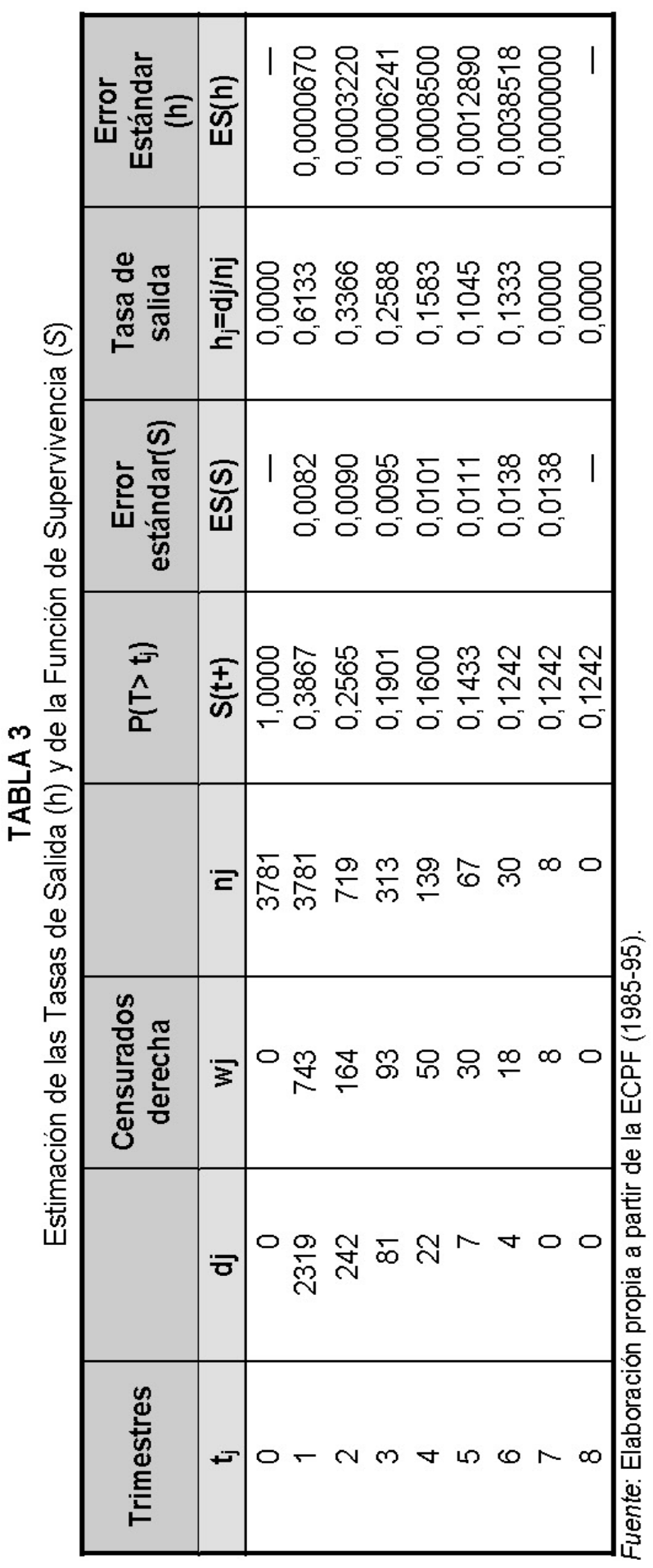


Para el análisis no paramétrico utilizamos 3.781 períodos de pobreza correspondientes a los períodos no censurados y censurados a la derecha. La estructura de la variable duración o permanencia en la pobreza expresada en trimestres para la muestra se recoge en la Tabla 3 , donde $n_{j}$ representa el número de hogares cuyo período de pobreza es mayor o igual que $t_{j}$ (número de trimestres); $d_{j}$ es el número de hogares que abandonan la pobreza en $t_{j}$ y $w_{j}$ es el número de datos censurados a la derecha en $t_{j .}{ }^{13}$ A partir de estos datos se ha estimado la función de supervivencia (ver Figura 4), la tasa de salida o de riesgo $h(t)$, así como sus respectivos errores estándar.

FIGURA 4

Estimación Kaplan-Meier de la Función de Supervivencia.

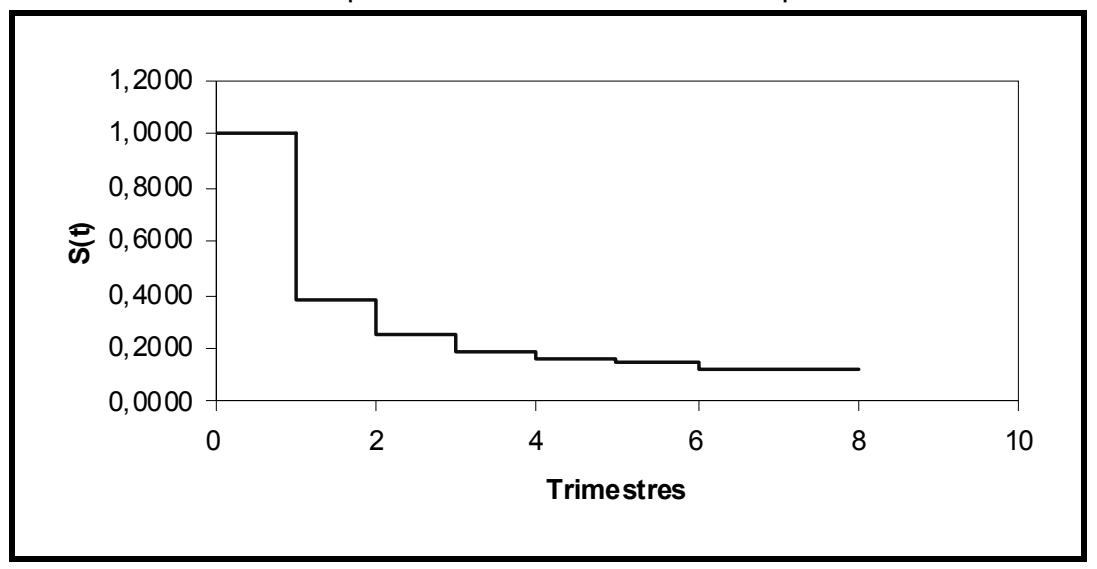

La Tabla 3 muestra que el $38,67 \%$ de los períodos de la muestra tienen duración de más de un trimestre, reduciéndose a un $25,65 \%$ si la duración en pobreza es de más dos trimestres y a un $16 \%$ si la duración en pobreza es mayor a cuatro trimestres. En cuanto a la tasa de salida de la pobreza, se observa que si el hogar está en situación de pobreza en el primer trimestre, la probabilidad de salir de la pobreza es del $61,33 \%$, mientras que si, por el contrario, se encuentra en el cuarto trimestre la probabilidad de abandonar el estado de pobreza se reduce a un $15,83 \%$. La tasa de salida es decreciente, lo que implica la existencia de dependencia de duración negativa a partir del primer trimestre, es decir, a medida que transcurre el tiempo disminuye la probabilidad de salir de la pobreza. Este fenómeno vendría explicado desde un punto vista social, porque a mayor tiempo del hogar en pobreza mayor será el esfuerzo necesario para salir de ella. Destacamos que a partir del segundo trimestre la probabilidad de salir de la pobreza continúa decreciendo en el tiempo de una forma más suave; sin embargo, la probabilidad de dejar la pobreza entre el primer y segundo trimestre parece decaer más rápidamente.

\footnotetext{
${ }^{13}$ De este modo, la única información que se dispone para los censurados a la derecha es que su período de permanencia en la pobreza es mayor que $t_{j}$.
} 
A continuación se analiza cómo afectan ciertas características relacionadas con el hogar al proceso de salida de la pobreza, para ello, se compara la función de supervivencia y riesgo de los hogares con distintas características. El objetivo que se persigue es doble: en primer lugar, comprobar cómo ciertas variables relacionadas con el sustentador principal (género, nivel educativo y situación laboral) afectan al proceso de salida de la pobreza, ${ }^{14} \mathrm{y}$ en segundo lugar, vamos a observar cómo ciertas variables relacionadas con el hogar (número de preceptores de ingresos y tamaño del municipio en el que reside el hogar) inciden en la duración en situación de pobreza económica. ${ }^{15}$

\section{Variable género}

En la Tabla 4 se realizan las estimaciones de las tasas de salida de la pobreza y la función de supervivencia (ver Figura 5) según género del sustentador principal.

\section{TABLA 4}

Estimaciones de la Tasa de Salida $(h)$ y la Función de Supervivencia $(S)$ según género del Sustentador Principal.

\begin{tabular}{|r|r|r|r|r|r|r|r|}
\hline \multicolumn{9}{|c|}{ Hombre } \\
\hline & \multicolumn{1}{|c|}{$\mathbf{d j}$} & $\mathbf{w j}$ & \multicolumn{1}{c|}{$\mathbf{n j}$} & $\mathbf{S}(\mathbf{t}+\mathbf{)}$ & $\mathbf{E S ( S )}$ & $\mathbf{h}(\mathbf{t})$ & $\mathbf{E S ( h )}$ \\
\hline 0 & 0 & 0 & 3111 & 1,0000 & - & 0,0000 & \\
1 & 1896 & 612 & 3111 & 0,3905 & 0,0087 & 0,6095 & 0,0087 \\
2 & 209 & 142 & 603 & 0,2552 & 0,0095 & 0,3466 & 0,0194 \\
3 & 63 & 78 & 252 & 0,1914 & 0,0100 & 0,2500 & 0,0273 \\
4 & 18 & 40 & 111 & 0,1604 & 0,0107 & 0,1622 & 0,0350 \\
5 & 7 & 25 & 53 & 0,1392 & 0,0119 & 0,1321 & 0,0465 \\
6 & 2 & 13 & 21 & 0,1259 & 0,0140 & 0,0952 & 0,0641 \\
7 & 0 & 6 & 6 & 0,1259 & 0,0140 & 0,0000 & 0,0000 \\
8 & 0 & 0 & 0 & 0,1259 & - & 0,0000 & - \\
\hline
\end{tabular}

\begin{tabular}{|r|r|r|r|r|r|r|r|}
\hline \multicolumn{8}{|c|}{ Mujer } \\
\hline & \multicolumn{1}{|c|}{$\mathbf{d j}$} & $\mathbf{w j}$ & \multicolumn{1}{c|}{$\mathbf{n j}$} & $\mathbf{S}(\mathbf{t}+\mathbf{)}$ & $\mathbf{E S ( S )}$ & $\mathbf{h}(\mathbf{t})$ & $\mathbf{E S ( h )}$ \\
\hline 0 & 0 & 0 & 667 & 1,0000 & - & 0,0000 & - \\
1 & 423 & 131 & 667 & 0,3658 & 0,0087 & 0,6342 & 0,0087 \\
2 & 33 & 20 & 113 & 0,2590 & 0,0095 & 0,2920 & 0,0194 \\
3 & 18 & 15 & 60 & 0,1813 & 0,0100 & 0,3000 & 0,0273 \\
4 & 4 & 10 & 27 & 0,1544 & 0,0107 & 0,1481 & 0,0350 \\
5 & 0 & 4 & 13 & 0,1544 & 0,0119 & 0,0000 & 0,0465 \\
6 & 2 & 5 & 9 & 0,1201 & 0,0140 & 0,2222 & 0,0641 \\
7 & 0 & 2 & 2 & 0,1201 & 0,0140 & 0,0000 & 0,0000 \\
8 & 0 & 0 & 0 & 0,1201 & - & 0,0000 & - \\
\hline
\end{tabular}

Fuente: Elaboración propia a partir de la ECPF (1985-95).

\footnotetext{
${ }^{14}$ Dado que el nivel educativo y la situación laboral pueden variar en los ocho trimestres y, por tanto, en el período de pobreza, hemos optado por elegir la modalidad que presentaba en la fecha de entrada en la pobreza

${ }^{15}$ Se ha optado por medir también estas variables en el momento de entrada en la pobreza.
} 
De la Tabla 4 se observa que no hay importantes diferencias en la duración en situación de pobreza económica entre aquellos hogares cuyo sustentador principal es hombre al de aquellos en los que es mujer. Así, aproximadamente el 39\% y el $37 \%$ de los hogares con sustentador principal hombre y mujer respectivamente permanece al menos un trimestre. Este porcentaje se reduce aproximadamente a un $25,52 \%$ y 25,90 para hombres y mujeres respectivamente, en el caso de que la permanencia en pobreza sea de más de dos trimestres. Las diferencias en ambos sexos es muy pequeña en aquellos casos en los que la permanencia en pobreza es superior o igual a 4 trimestres. En principio y a raíz de los resultados, se podría pensar que el "fenómeno de feminización" de la pobreza observado en los estudios de corte transversal, se observa en el estudio longitudinal aunque no de una forma tan pronunciada principalmente para períodos muy cortos de pobreza (dos trimestres consecutivos). No obstante, hemos aplicado un "test de log-rank"16 [Hosmer y Lemeshow (1999)] para contrastar si existen diferencias significativas en las funciones de supervivencia, concluyéndose que el género influye significativamente en la duración del estado de pobreza.

\section{FIGURA 5}

Estimación Kaplan-Meier de la Función de Supervivencia según género del sustentador principal.

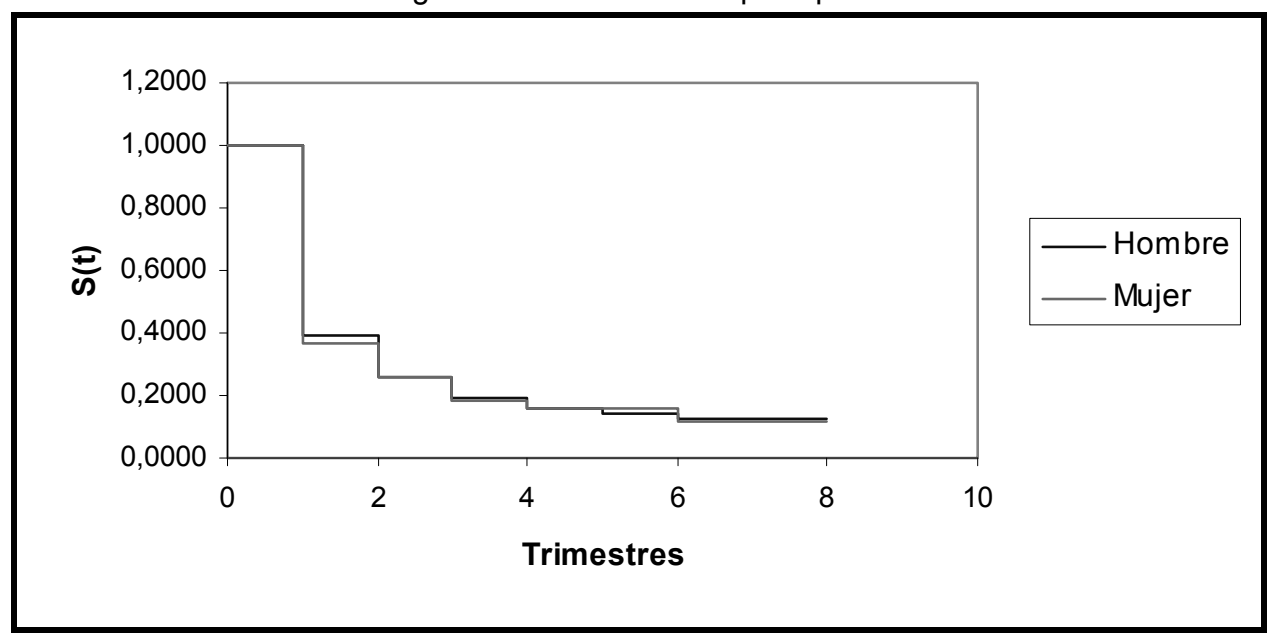

\section{Variable nivel educativo}

El nivel de educación ha sido codificado en cuatro categorías. "Estudios de Primer Nivel" (ED1), en este nivel educativo hemos agrupado la categoría de Analfabetos y la categoría Sin Estudios, definida en la metodología de la ECPF. En la categoría,

\footnotetext{
${ }^{16}$ El valor del estadístico Log-rank ha sido de 54,78 con un p-valor asociado menor del $1 \%$, por lo que se rechazaría la hipótesis nula de igualdad de las funciones de supervivencia.
} 
"Estudios de Segundo Nivel" (ED2) se han agrupado la Enseñanza de Primer Grado y la Enseñanza de Segundo Grado primer ciclo. La categoría "Estudios de Tercer Nivel" (ED3) ha sido creada agrupando la Enseñanza Segundo grado segundo ciclo. La última categoría "Estudios de Cuarto Nivel" (ED4) comprende la Enseñanza de Tercer Grado, primer ciclo y la Enseñanza de Tercer grado, segundo y tercer ciclo.

En la Tabla 5 se presentan las estimaciones de las tasa de salida y función de supervivencia (ver Figura 6) para las sub-muestras relativas a diferentes niveles educativos del sustentador principal en el momento en que empezaba el período de pobreza.

TABLA 5

Estimaciones de las Tasa de Salida y Función de Supervivencia según nivel educativo del sustentador principal.

\begin{tabular}{|rrrrrrrr|}
\hline \multicolumn{7}{|c|}{ Estudios de Primer Nivel } \\
\hline & dj & wj & \multicolumn{1}{c|}{$\mathbf{j}$} & $\mathbf{S}(\mathbf{t}+)$ & $\mathbf{E S}(\mathbf{S})$ & $\mathbf{h}(\mathbf{t})$ & $\mathbf{E S}(\mathbf{h})$ \\
\hline 0 & 0 & 0 & 1110 & 1,0000 & - & 0,0000 & - \\
1 & 556 & 275 & 1110 & 0,4991 & 0,0150 & 0,5009 & 0,0367 \\
2 & 89 & 63 & 279 & 0,3399 & 0,0173 & 0,3190 & 0,1056 \\
3 & 33 & 37 & 127 & 0,2516 & 0,0184 & 0,2598 & 0,1789 \\
4 & 8 & 24 & 57 & 0,2163 & 0,0196 & 0,1404 & 0,3536 \\
5 & 2 & 9 & 25 & 0,1990 & 0,0215 & 0,0800 & 0,0000 \\
6 & 3 & 9 & 14 & 0,1563 & 0,0276 & 0,2143 & - \\
7 & 0 & 2 & 2 & 0,1563 & 0,0276 & 0,0000 & - \\
8 & 0 & 0 & 0 & 0,1563 & - & 0,0000 & - \\
\hline
\end{tabular}

\begin{tabular}{|rrrrrrr|}
\hline \multicolumn{7}{|c|}{ Estudios de Sequndo Nivel } \\
\hline di & wi & \multicolumn{1}{c|}{$\mathbf{n j}$} & $\mathbf{S}(\mathbf{t}+)$ & $\mathbf{E S ( S )}$ & $\mathbf{h}(\mathbf{t}) \mathbf{i}$ & $\mathbf{E S ( h )}$ \\
\hline 0 & 0 & 2413 & 1,0000 & - & 0,0000 & - \\
1571 & 427 & 2413 & 0,3489 & 0,0097 & 0,6511 & 0,0097 \\
145 & 90 & 415 & 0,2270 & 0,0103 & 0,3494 & 0,0234 \\
47 & 54 & 180 & 0,1677 & 0,0106 & 0,2611 & 0,0327 \\
13 & 25 & 79 & 0,1401 & 0,0113 & 0,1646 & 0,0417 \\
5 & 20 & 41 & 0,1231 & 0,0123 & 0,1220 & 0,0511 \\
1 & 9 & 16 & 0,1154 & 0,0137 & 0,0625 & 0,0605 \\
0 & 6 & 6 & 0,1154 & 0,0137 & 0,0000 & 0,0000 \\
0 & 0 & 0 & 0,1154 & - & 0,0000 & - \\
\hline
\end{tabular}


TABLA 5 (continuación)

Estimaciones de las Tasa de Salida y Función de Supervivencia según nivel educativo del sustentador principal.

\begin{tabular}{|rrrrrrrr|}
\hline \multicolumn{7}{|c|}{ Estudios de Tercer Nivel } \\
\hline $\mathbf{t j}$ & $\mathbf{d j}$ & $\mathbf{w j}$ & $\mathbf{n j}$ & $\mathbf{S}(\mathbf{t}+\mathbf{)}$ & $\mathbf{E S ( S )}$ & $\mathbf{h}(\mathbf{t})$ & ES(h) \\
\hline 0 & 0 & 0 & 155 & 1,0000 & - & 0,0000 & - \\
1 & 109 & 28 & 155 & 0,2968 & 0,0367 & 0,7032 & 0,0367 \\
2 & 5 & 8 & 18 & 0,2143 & 0,0410 & 0,2778 & 0,1056 \\
3 & 1 & 2 & 5 & 0,1715 & 0,0505 & 0,2000 & 0,1789 \\
4 & 1 & 0 & 2 & 0,0857 & 0,0657 & 0,5000 & 0,3536 \\
5 & 0 & 1 & 1 & 0,0857 & 0,0657 & 0,0000 & 0,0000 \\
6 & 0 & 0 & 0 & 0,0857 & - & 0,0000 & - \\
7 & 0 & 0 & 0 & 0,0857 & - & 0,0000 & - \\
8 & 0 & 0 & 0 & 0,0857 & - & 0,0000 & - \\
\hline
\end{tabular}

\begin{tabular}{|rrrrrrr|}
\hline \multicolumn{7}{|c|}{ Estudios Cuarto Nivel } \\
\hline $\mathbf{d j}$ & $\mathbf{w j}$ & $\mathbf{n j}$ & $\mathbf{S}(\mathbf{t}+)$ & $\mathbf{E S}(\mathbf{S})$ & $\mathbf{h}(\mathbf{t})$ & $\mathbf{E S}(\mathbf{h})$ \\
\hline 0 & 0 & 103 & 1,0000 & - & 0,0000 & - \\
83 & 13 & 103 & 0,1942 & 0,0390 & 0,8058 & 0,0390 \\
3 & 3 & 7 & 0,1110 & 0,0426 & 0,4286 & 0,1870 \\
0 & 0 & 1 & 0,1110 & 0,0426 & 0,0000 & 0,0000 \\
0 & 1 & 1 & 0,1110 & 0,0426 & 0,0000 & 0,0000 \\
0 & 0 & 0 & 0,1110 & - & 0,0000 & - \\
0 & 0 & 0 & 0,1110 & - & 0,0000 & - \\
0 & 0 & 0 & 0,1110 & - & 0,0000 & - \\
0 & 0 & 0 & 0,1110 & - & 0,0000 & - \\
\hline
\end{tabular}

Fuente: Elaboración propia a partir de la ECPF (1985-95).

FIGURA 6

Estimación Kaplan-Meier de la Función de Supervivencia según nivel educativo del sustentador principal.

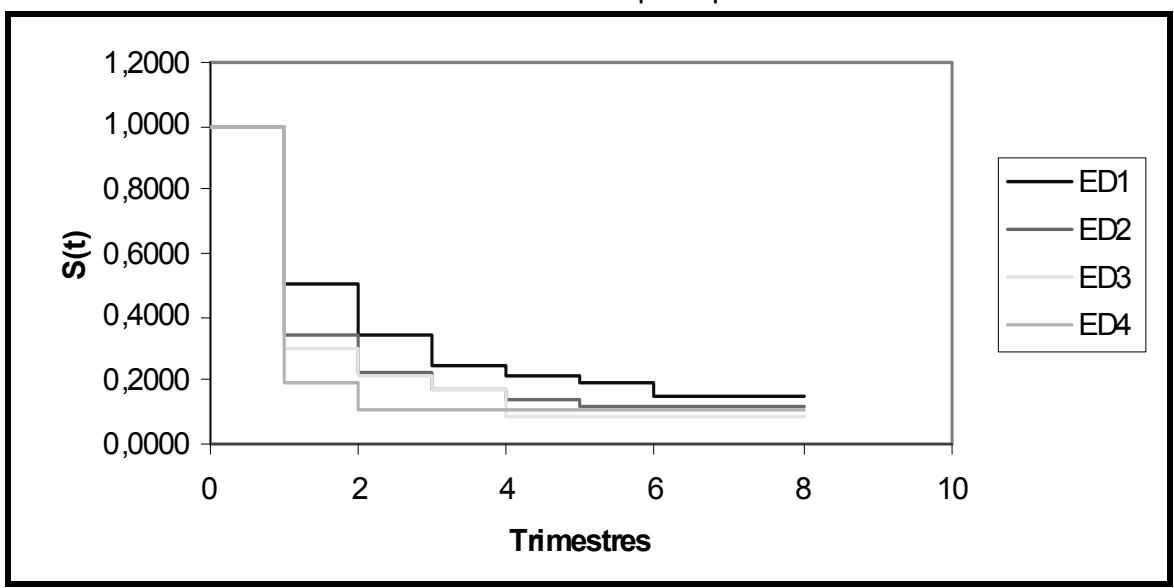


Atendiendo a la Tabla 5 se puede observar que las duraciones en pobreza son más cortas cuanto más elevado es el nivel de estudios (ED3 o ED4) ${ }^{17}$. Así, mientras que el $21,63 \%$ y $14,01 \%$ de los hogares, cuyo sustentador principal tiene educación ED1 o ED2, tienen períodos de pobreza de más de cuatro trimestres, este porcentaje se reduce al $8,87 \%$ y $11 \%$ en aquellos hogares en los que el sustentador pertenecen al nivel educativo ED3 y ED4 respectivamente.

La Tabla 5 permite también observar como la salida de la pobreza en el primer trimestre es muy elevada para los hogares cuyo sustentador principal posee estudios de tercer y cuarto nivel $(70,32 \%$ y $80,58 \%$ respectivamente). No obstante, todo parece indicar que estos hogares tienen más dificultades en salir de la pobreza que aquellos hogares cuyo sustentador principal tiene inferior cualificación cuanto mayor es el tiempo de permanencia en la misma. Este último resultado parece indicar que aquellos que tienen formación profesional tienden a salir más rápidamente de dificultades económicas que aquellos que cursaron estudios de bachillerato o universitarios debido fundamentalmente a las exigencias profesionales de estos colectivos.

\section{Situación Laboral}

La situación laboral ha sido codificada en seis modalidades: "Activos que trabajan por cuenta propia" (SL1), "Asalariados Cualificados" (SL2), "Asalariados agrarios sin especializar" (SL3), "Asalariados no agrarios sin especializar" (SL4), "Parados que buscan empleo" (SL5) y "No activos" (SL6). Los resultados de la comparación de las sub-muestras según la situación laboral del sustentador principal aparecen recogidos en la Tabla 6 .

TABLA 6

Estimaciones de las Tasa de Salida y Función de Supervivencia según Situación Laboral del sustentador principal.

\begin{tabular}{|rrrrrrrr|}
\hline \multicolumn{7}{|c|}{ Activos que trabajan por cuenta propia } \\
\hline $\mathbf{T}$ & $\mathbf{d j}$ & $\mathbf{W j}$ & \multicolumn{1}{c|}{$\mathbf{n j}$} & $\mathbf{S}(\mathbf{t}+)$ & $\mathbf{E S}(\mathbf{S})$ & $\mathbf{h}(\mathbf{t})$ & ES(h) \\
\hline 0 & 0 & 0 & 1270 & 1,00000 & - & 0,0000 & - \\
1 & 899 & 192 & 1270 & 0,29213 & 0,0128 & 0,7079 & 0,0128 \\
2 & 67 & 40 & 179 & 0,18278 & 0,0132 & 0,3743 & 0,0362 \\
3 & 25 & 22 & 72 & 0,11932 & 0,0134 & 0,3472 & 0,0561 \\
4 & 7 & 8 & 25 & 0,08591 & 0,0144 & 0,2800 & 0,0898 \\
5 & 2 & 4 & 10 & 0,06873 & 0,0159 & 0,2000 & 0,1265 \\
6 & 1 & 1 & 4 & 0,05154 & 0,0190 & 0,2500 & 0,2165 \\
7 & 0 & 2 & 2 & 0,05154 & 0,0190 & 0,0000 & 0,0000 \\
8 & 0 & 0 & 0 & 0,05154 & - & 0,0000 & - \\
\hline
\end{tabular}

\footnotetext{
${ }^{17}$ Este hecho puede también observarse en la Figura 6. Dado que hemos definido la duración como el tiempo de permanencia en pobreza, al comparar dos curvas de supervivencia aquella que se situé por debajo indica que el grupo representado por dicha curva permanece menos tiempo en pobreza que aquel que se sitúe por arriba. Así, la Figura 6 muestra que en términos generales las curvas de supervivencias para los hogares cuyo sustentador principal tiene educación superior ED3 y ED4, está por debajo de las otras curvas que representan menor nivel educativo.
} 
TABLA 6 (continuación)

Estimaciones de las Tasa de Salida y Función de Supervivencia según Situación Laboral del sustentador principal.

\begin{tabular}{|rrrrrrr|}
\hline \multicolumn{7}{|c|}{ Asalariados Cualificados } \\
\hline $\mathbf{d j}$ & $\mathbf{w j}$ & \multicolumn{1}{c|}{$\mathbf{n j}$} & $\mathbf{S}(\mathbf{t}+\mathbf{)}$ & $\mathbf{E S}(\mathbf{S})$ & $\mathbf{h}(\mathbf{t})$ & $\mathbf{E S}(\mathbf{h})$ \\
\hline 0 & 0 & 588 & 1,0000 & - & 0,0000 & - \\
395 & 108 & 588 & 0,3282 & 0,0194 & 0,6718 & 0,0194 \\
28 & 25 & 85 & 0,2201 & 0,0212 & 0,3294 & 0,0510 \\
10 & 9 & 32 & 0,1513 & 0,0232 & 0,3125 & 0,0819 \\
3 & 5 & 13 & 0,1164 & 0,0251 & 0,2308 & 0,1169 \\
0 & 3 & 5 & 0,1164 & 0,0251 & 0,0000 & 0,0000 \\
0 & 1 & 2 & 0,1164 & 0,0251 & 0,0000 & 0,0000 \\
0 & 1 & 1 & 0,1164 & 0,0251 & 0,0000 & 0,0000 \\
0 & 0 & 0 & 0,1164 & - & 0,0000 & - \\
\hline
\end{tabular}

\begin{tabular}{|rrrrrrrr|}
\hline \multicolumn{7}{|c|}{ Asalariados Agrarios sin especializar } \\
\hline $\mathbf{T}$ & $\mathbf{d j}$ & $\mathbf{W j}$ & \multicolumn{1}{c}{$\mathbf{n j}$} & $\mathbf{S}(\mathbf{t}+\mathbf{)}$ & $\mathbf{E S}(\mathbf{S})$ & $\mathbf{h}(\mathbf{t})$ & ES(h) \\
\hline 0 & 0 & 0 & 123 & 1,0000 & - & 0,0000 & - \\
1 & 47 & 37 & 123 & 0,6179 & 0,0438 & 0,3821 & 0,0438 \\
2 & 7 & 9 & 39 & 0,5070 & 0,0523 & 0,1795 & 0,0615 \\
3 & 4 & 7 & 23 & 0,4188 & 0,0589 & 0,1739 & 0,0790 \\
4 & 4 & 2 & 12 & 0,2792 & 0,0692 & 0,3333 & 0,1361 \\
5 & 0 & 5 & 6 & 0,2792 & 0,0692 & 0,0000 & 0,0000 \\
6 & 0 & 1 & 1 & 0,2792 & 0,0692 & 0,0000 & 0,0000 \\
7 & 0 & 0 & 0 & 0,2792 & - & 0,0000 & - \\
8 & 0 & 0 & 0 & 0,2792 & - & 0,0000 & - \\
\hline
\end{tabular}

\begin{tabular}{|rrrrrrr|}
\hline \multicolumn{7}{|c|}{ Asalariados No agrarios sin especializar } \\
\hline $\mathbf{d j}$ & $\mathbf{w j}$ & $\mathbf{n j}$ & $\mathbf{S}(\mathbf{t}+)$ & $\mathbf{E S}(\mathbf{S})$ & $\mathbf{h}(\mathbf{t}) \mathbf{j}$ & $\mathbf{E S ( h )}$ \\
\hline 0 & 0 & 322 & 1,0000 & - & 0,0000 & - \\
180 & 68 & 322 & 0,4410 & 0,0277 & 0,5590 & 0,0277 \\
23 & 15 & 74 & 0,3039 & 0,0304 & 0,3108 & 0,0538 \\
8 & 13 & 36 & 0,2364 & 0,0317 & 0,2222 & 0,0693 \\
3 & 7 & 15 & 0,1891 & 0,0352 & 0,2000 & 0,1033 \\
0 & 4 & 5 & 0,1891 & 0,0352 & 0,0000 & 0,0000 \\
0 & 1 & 1 & 0,1891 & 0,0352 & 0,0000 & 0,0000 \\
0 & 0 & 0 & 0,1891 & - & 0,0000 & - \\
0 & 0 & 0 & 0,1891 & - & 0,0000 & - \\
\hline
\end{tabular}


TABLA 6 (continuación)

Estimaciones de las Tasa de Salida y Función de Supervivencia según Situación Laboral del sustentador principal.

\begin{tabular}{|rrrrrrrr|}
\hline \multicolumn{7}{|c|}{ Parados que buscan empleo } \\
\hline $\mathbf{T}$ & $\mathbf{d j}$ & $\mathbf{W j}$ & \multicolumn{1}{c|}{$\mathbf{j}$} & $\mathbf{S}(\mathbf{t}+)$ & $\mathbf{E S}(\mathbf{S})$ & $\mathbf{h}(\mathbf{t}) \mathbf{j}$ & $\mathbf{E S ( h )}$ \\
\hline 0 & 0 & 0 & 401 & 1,0000 & - & 0,0000 & - \\
1 & 158 & 91 & 401 & 0,6060 & 0,0244 & 0,3940 & 0,0244 \\
2 & 57 & 34 & 152 & 0,3787 & 0,0283 & 0,3750 & 0,0393 \\
3 & 14 & 19 & 61 & 0,2918 & 0,0298 & 0,2295 & 0,0538 \\
4 & 0 & 12 & 28 & 0,2918 & 0,0298 & 0,0000 & 0,0000 \\
5 & 3 & 3 & 16 & 0,2371 & 0,0374 & 0,1875 & 0,0976 \\
6 & 0 & 7 & 10 & 0,2371 & 0,0374 & 0,0000 & 0,0000 \\
7 & 0 & 3 & 3 & 0,2371 & 0,0374 & 0,0000 & 0,0000 \\
8 & 0 & 0 & 0 & 0,2371 & - & 0,0000 & - \\
\hline
\end{tabular}

\begin{tabular}{|rrrrrrr|}
\hline \multicolumn{7}{|c|}{ Asalariados No activos } \\
\hline dj & wj & \multicolumn{1}{c|}{$\mathbf{n j}$} & $\mathbf{S}(\mathbf{t}+)$ & $\mathbf{E S}(\mathbf{S})$ & $\mathbf{h}(\mathbf{t})$ & $\mathbf{E S}(\mathbf{h})$ \\
\hline 0 & 0 & 1077 & 1,0000 & - & 0,0000 & - \\
640 & 247 & 1077 & 0,4058 & 0,0196 & 0,5942 & 0,020 \\
60 & 41 & 190 & 0,2776 & 0,0224 & 0,3158 & 0,033 \\
20 & 23 & 89 & 0,2152 & 0,0246 & 0,2247 & 0,041 \\
5 & 16 & 46 & 0,1918 & 0,0270 & 0,1087 & 0,050 \\
2 & 11 & 25 & 0,1765 & 0,0327 & 0,0800 & 0,086 \\
3 & 7 & 12 & 0,1324 & 0,0414 & 0,2500 & 0,132 \\
0 & 2 & 2 & 0,1324 & 0,0414 & 0,0000 & 0,000 \\
0 & 0 & 0 & 0,1324 & - & 0,0000 & - \\
\hline
\end{tabular}

Fuente: Elaboración propia a partir de la ECPF 1985-95.

En la Tabla 6 se observa que si atendemos a la probabilidad de permanecer en pobreza más de cuatro trimestres, observamos como los activos que trabajan por cuentan propia son los que tienen menor probabilidad de permanecer más tiempo en pobreza económica. Así, el 8,891\% de los hogares con sustentador principal trabajando por cuenta propia permanece más de 4 trimestres consecutivos en pobreza, frente al $29,18 \%$ en el caso de los parados y el $27,92 \%$ en el caso de los asalariados agrarios. Destacar, que para períodos de pobreza superiores a cinco trimestres, el hecho de que la proporción estimada de hogares pobres cuyo sustentador es parado que busca empleo, $23,71 \%$, sea menor que la proporción de hogares pobres cuyo sustentador principal pertenece a la categoría laboral, Asalariados agrarios sin especializar, con un $27,92 \%$ puede ser debido al efecto que pueda tener la protección por desempleo en aquellos hogares cuyo sustentador es un parado que busca empleo.

Si atendemos a la estimación de la función riesgo o tasa de salida, se observa que en términos generales es decreciente en el tiempo, es decir, la probabilidad de salir de la situación de pobreza económica es menor cuanto más tiempo permanece 
el hogar en ese estado. La probabilidad de salir de la situación de pobreza económica en el primer trimestre es superior al $50 \%$ en las categorías correspondientes a Activos que trabajan por cuenta propia (70,79\%), Asalariados cualificados $(67,18 \%)$ y Asalariados no agrarios sin especializar $(55,90 \%)$. Asimismo, la probabilidad de salir después de 6 trimestres consecutivos en pobreza es nula para todas las categorías.

\section{Perceptores de Ingreso $^{18}$}

El número de perceptores de ingresos ha sido codificado en cuatro modalidades: Un perceptor de ingresos (PI1), dos perceptores de ingresos (PI2), tres perceptores de ingresos (PI3) y cuatro perceptores o más (PI4).

De acuerdo con las estimaciones obtenidas en la Tabla 7, el número de perceptores de ingresos es, de hecho, una variable que incide en el tiempo de permanencia en pobreza. Se observa que mientras el $13,83 \%$ de los hogares con cuatro o más preceptores tiene una duración en pobreza superior a 5 trimestres, este porcentaje se eleva a $22,81 \%$ y $56,43 \%$ para las sub-muestras de dos perceptores y un preceptor de ingresos respectivamente. Hemos de destacar que las tasas de riesgo o probabilidad de salida de la pobreza en cada sub-muestra son mayores para los hogares donde el número de preceptores es mayor. Así, la probabilidad de salir de la pobreza en el primer trimestre es del $62,78 \%$ para los hogares con cuatro o más perceptores de ingresos, del 65,89\% para aquellos con 3 perceptores, del $44,68 \%$ para hogares con dos preceptores de ingreso, y reduciéndose a $31,03 \%$ en el caso de un preceptor de ingresos. Destacar que la probabilidad de salir de la pobreza a partir del tercer trimestre es cero para los hogares con un único preceptor de ingreso.

\footnotetext{
${ }^{18}$ Se ha estimado la función de supervivencia y la función riesgo para la variable tamaño del hogar. No se observa un comportamiento sistemático para las distintas curvas de supervivencia, es decir, estas van intercambiando sus posiciones para las distintas sub-muestras según las duraciones. Este hecho puede ser debido a que esta variable interacciona de alguna manera con el número de perceptores de ingresos de ahí la confusa incidencia del tamaño del hogar sobre la duración de la pobreza. Ante este hecho, analizamos cómo incide el número de perceptores del ingreso en la duración en pobreza.
} 
TABLA 7

Estimaciones de las Tasa de Salida y Función de Supervivencia según Número de Preceptores de Ingreso.

\begin{tabular}{|rcrrrrrr|}
\hline \multicolumn{7}{|c|}{ Un Preceptor de Inqreso } \\
\hline $\mathbf{T}$ & $\mathbf{d j}$ & $\mathbf{w j}$ & $\mathbf{n j}$ & $\mathbf{S}(\mathbf{t}+\mathbf{)}$ & $\mathbf{E S ( S )}$ & $\mathbf{h}(\mathbf{t})$ & $\mathbf{E S ( h )}$ \\
\hline 0 & 0 & 0 & 29 & 1,0000 & 0,0933 & - & 0,0933 \\
1 & 9 & 9 & 29 & 0,6897 & 0,0933 & 0,3103 & 0,0000 \\
2 & 2 & 6 & 11 & 0,5643 & 0,0933 & 0,1818 & 0,0000 \\
3 & 0 & 3 & 4 & 0,5643 & 0,0933 & 0,0000 & 0,0000 \\
4 & 0 & 0 & 1 & 0,5643 & - & 0,0000 & 0,0000 \\
5 & 0 & 0 & 1 & 0,5643 & - & 0,0000 & - \\
6 & 0 & 0 & 0 & 0,5643 & - & 0,0000 & - \\
7 & 0 & 0 & 0 & 0,5643 & - & 0,0000 & - \\
8 & 0 & 0 & 0 & 0,5643 & - & 0,0000 & - \\
\hline
\end{tabular}

\begin{tabular}{|rrrrrrr|}
\hline \multicolumn{7}{|c|}{ Dos Preceptores de Ingreso } \\
\hline $\mathbf{d j}$ & $\mathbf{w j}$ & $\mathbf{n j}$ & $\mathbf{S}(\mathbf{t}+\mathbf{)}$ & $\mathbf{E S}(\mathbf{S})$ & $\mathbf{h}(\mathbf{t})$ & $\mathbf{E S}(\mathbf{h})$ \\
\hline 0 & 0 & 611 & 1,0000 & 0,0231 & - & 0,02309 \\
273 & 169 & 611 & 0,5532 & 0,0289 & 0,4468 & 0,04142 \\
60 & 39 & 169 & 0,3568 & 0,0311 & 0,3550 & 0,05786 \\
18 & 17 & 70 & 0,2650 & 0,0323 & 0,2571 & 0,05040 \\
3 & 15 & 35 & 0,2423 & 0,0323 & 0,0857 & 0,00000 \\
1 & 6 & 17 & 0,2281 & 0,0323 & 0,0588 & 0,00000 \\
0 & 6 & 10 & 0,2281 & 0,0323 & 0,0000 & 0,00000 \\
0 & 4 & 4 & 0,2281 & - & 0,0000 & - \\
0 & 0 & 0 & 0,2281 & - & 0,0000 & - \\
\hline
\end{tabular}

\begin{tabular}{|rrrrrrrr|}
\hline \multicolumn{8}{|c|}{ Tres Preceptores de Ingreso } \\
\hline $\mathbf{T}$ & \multicolumn{1}{c}{$\mathbf{d j}$} & $\mathbf{w j}$ & \multicolumn{1}{c|}{$\mathbf{n j}$} & $\mathbf{S}(\mathbf{t}+)$ & $\mathbf{E S}(\mathbf{S})$ & $\mathbf{h}(\mathbf{t})$ & $\mathbf{E S ( h )}$ \\
\hline 0 & 0 & 0 & 2096 & 1,0000 & 0,0167 & - & 0,0167 \\
1 & 1381 & 361 & 2096 & 0,3411 & 0,0198 & 0,6589 & 0,0280 \\
2 & 119 & 76 & 354 & 0,2265 & 0,0223 & 0,3362 & 0,0428 \\
3 & 46 & 47 & 159 & 0,1609 & 0,0243 & 0,2893 & 0,0517 \\
4 & 12 & 21 & 66 & 0,1317 & 0,0262 & 0,1818 & 0,0512 \\
5 & 3 & 14 & 33 & 0,1197 & 0,0383 & 0,0909 & 0,1323 \\
6 & 3 & 10 & 16 & 0,0973 & - & 0,1875 & - \\
7 & 0 & 3 & 3 & 0,0973 & - & 0,0000 & - \\
8 & 0 & 0 & 0 & 0,0973 & - & 0,0000 & - \\
\hline
\end{tabular}


TABLA 7 (continuación)

Estimaciones de las Tasa de Salida y Función de Supervivencia según Número de Preceptores de Ingreso.

\begin{tabular}{|rrrrrrr|}
\hline \multicolumn{7}{|c|}{ Cuatro o más Perceptores } \\
\hline $\mathbf{d j}$ & $\mathbf{w j}$ & \multicolumn{1}{c}{$\mathbf{n j}$} & $\mathbf{S}(\mathbf{t}+\mathbf{)}$ & $\mathbf{E S ( S )}$ & $\mathbf{h}(\mathbf{t}) \mathbf{j}$ & $\mathbf{E S}(\mathbf{h})$ \\
\hline 0 & 0 & 1045 & 1,0000 & 0,0215 & - & 0,0215 \\
656 & 204 & 1045 & 0,3722 & 0,0261 & 0,6278 & 0,0396 \\
62 & 43 & 185 & 0,2475 & 0,0286 & 0,3351 & 0,0504 \\
17 & 26 & 80 & 0,1949 & 0,0332 & 0,2125 & 0,0800 \\
7 & 14 & 37 & 0,1580 & 0,0376 & 0,1892 & 0,0949 \\
2 & 10 & 16 & 0,1383 & 0,0786 & 0,1250 & 0,3536 \\
1 & 2 & 4 & 0,1037 & - & 0,2500 & - \\
0 & 1 & 1 & 0,1037 & - & 0,0000 & - \\
0 & 0 & 0 & 0,1037 & - & 0,0000 & - \\
\hline
\end{tabular}

\section{Tamaño del Municipio}

Por último, hemos realizado un análisis de las sub-muestras según el tamaño del municipio donde se encuentra localizado el hogar. El tamaño del municipio ha sido codificado atendiendo a cuatro modalidades. TM1, hasta 10.000 habitantes. TM2, de 10.001 a 50.000 . TM3, de 50.001 a 500.000 y capitales menores. TM4, de más de 500001 habitantes.

Las estimaciones de las funciones de supervivencia y tasas de salida para las distintas categorías de la variable Tamaño del Municipio se recogen ${ }^{19}$ en la Tabla 8 , en la cual se observa que la probabilidad de salir de la pobreza en el primer trimestre es mayor para aquellos hogares que habitan en municipios de más de 50.001 habitantes (un 64,76\% para TM3 y 65,05\% para TM4). No obstante, esta probabilidad también es bastante elevada para las categorías TM1 y TM2 $(59,84 \%$ y $57,80 \%$, respectivamente). Destacamos que para los municipios de más de 500.000 habitantes la probabilidad de salir de la situación de pobreza económica es nula a partir del sexto trimestre.

\footnotetext{
${ }^{19}$ De la representación gráfica de la estimación Kaplan-Meier de las funciones de supervivencia para las sub-muestras correspondientes a las distintas categorías del tamaño del municipio se observa un comportamiento no sistemático. Este comportamiento no sistemático de las curvas de supervivencia también fue observado para las variables nivel educativo, categoría laboral del sustentador principal y número de perceptores de ingresos.
} 
TABLA 8

Estimaciones de las Tasa de Salida y Función de Supervivencia según

Tamaño del Municipio.

\begin{tabular}{|rrrrrrrr|}
\hline \multicolumn{8}{|c|}{ Hasta $\mathbf{1 0 . 0 0 0}$ habitantes } \\
\hline $\mathbf{T}$ & $\mathbf{d j}$ & $\mathbf{w j}$ & \multicolumn{1}{c|}{$\mathbf{n j}$} & $\mathbf{S}(\mathbf{t}+\mathbf{)}$ & $\mathbf{E S}(\mathbf{S})$ & $\mathbf{h}(\mathbf{t}) \mathbf{j}$ & $\mathbf{E S ( h )}$ \\
\hline 0 & 0 & 0 & 1387 & 1 & - & - & - \\
1 & 830 & 272 & 1387 & 0,4016 & 0,0178 & 0,5984 & 0,0178 \\
2 & 104 & 50 & 285 & 0,2550 & 0,0217 & 0,3649 & 0,0317 \\
3 & 33 & 43 & 131 & 0,1908 & 0,0230 & 0,2519 & 0,0421 \\
4 & 9 & 20 & 55 & 0,1596 & 0,0256 & 0,1636 & 0,0606 \\
5 & 4 & 13 & 26 & 0,1350 & 0,0274 & 0,1538 & 0,0540 \\
6 & 2 & 5 & 9 & 0,1050 & 0,0523 & 0,2222 & 0,2165 \\
7 & 0 & 2 & 2 & 0,1050 & - & 0,0000 & - \\
8 & 0 & 0 & 0 & 0,1050 & - & 0,0000 & - \\
\hline
\end{tabular}

\begin{tabular}{|rrrrrrr|}
\hline \multicolumn{7}{|c|}{ De $\mathbf{1 0 . 0 0 0} \mathbf{~} \mathbf{5 0 . 0 0 0}$ habitantes } \\
\hline $\mathbf{d j}$ & $\mathbf{w j}$ & $\mathbf{n j}$ & $\mathbf{S ( t + )}$ & $\mathbf{E S ( S )}$ & $\mathbf{h}(\mathbf{t})$ & $\mathbf{E S ( h )}$ \\
\hline 0 & 0 & 898 & 1 & - & - & - \\
519 & 197 & 898 & 0,4220 & 0,0222 & 0,5780 & 0,0222 \\
59 & 44 & 182 & 0,2852 & 0,0271 & 0,3242 & 0,0396 \\
29 & 20 & 79 & 0,1805 & 0,0313 & 0,3671 & 0,0672 \\
4 & 12 & 30 & 0,1565 & 0,0321 & 0,1333 & 0,0512 \\
0 & 8 & 14 & 0,1565 & 0,0321 & 0,0000 & 0,0000 \\
1 & 3 & 6 & 0,1304 & 0,0655 & 0,1667 & 0,2722 \\
0 & 2 & 2 & 0,1304 & - & 0,0000 & - \\
0 & 0 & 0 & 0,1304 & - & 0,0000 & - \\
\hline
\end{tabular}

\begin{tabular}{|rrrrrrrr|}
\hline \multicolumn{7}{|c|}{ De $\mathbf{5 0 . 0 0 1} \mathbf{a} \mathbf{5 0 0 . 0 0 0}$ habitantes } \\
\hline $\mathbf{T}$ & $\mathbf{d j}$ & \multicolumn{1}{c}{ wj } & \multicolumn{1}{c|}{$\mathbf{n j}$} & $\mathbf{S}(\mathbf{t}+)$ & $\mathbf{E S ( S )}$ & $\mathbf{h}(\mathbf{t})$ & ES(h) \\
\hline 0 & 0 & 0 & 1104 & 1 & - & - & - \\
1 & 715 & 201 & 1104 & 0,3524 & 0,0228 & 0,6476 & 0,0228 \\
2 & 57 & 54 & 188 & 0,2455 & 0,0269 & 0,3032 & 0,0374 \\
3 & 15 & 21 & 77 & 0,1977 & 0,0305 & 0,1948 & 0,0504 \\
4 & 6 & 15 & 41 & 0,1688 & 0,0326 & 0,1463 & 0,0471 \\
5 & 1 & 6 & 20 & 0,1603 & 0,0373 & 0,0500 & 0,0688 \\
6 & 1 & 9 & 13 & 0,1480 & 0,0373 & 0,0769 & 0,0000 \\
7 & 0 & 3 & 3 & 0,1480 & 0,0373 & 0,0000 & 0,0000 \\
8 & 0 & 0 & 0 & 0,1480 & - & 0,0000 & - \\
\hline
\end{tabular}


TABLA 8 (continuación)

Estimaciones de las Tasa de Salida y Función de Supervivencia según Tamaño del Municipio.

\begin{tabular}{|rrrrrrr|}
\hline \multicolumn{7}{|c|}{ Más de $\mathbf{5 0 0 . 0 0 0}$ habitantes } \\
\hline $\mathbf{d j}$ & $\mathbf{w j}$ & \multicolumn{1}{c}{$\mathbf{n j}$} & $\mathbf{S}(\mathbf{t}+\mathbf{+}$ & $\mathbf{E S}(\mathbf{S})$ & $\mathbf{h}(\mathbf{t})$ & $\mathbf{E S ( h )}$ \\
\hline 0 & 0 & 392 & 1 & - & - & - \\
255 & 73 & 392 & 0,3495 & 0,0402 & 0,6505 & 0,0402 \\
22 & 16 & 64 & 0,2294 & 0,0489 & 0,3438 & 0,0676 \\
4 & 9 & 26 & 0,1941 & 0,0532 & 0,1538 & 0,0798 \\
3 & 3 & 13 & 0,1493 & 0,0661 & 0,2308 & 0,1571 \\
2 & 3 & 7 & 0,1066 & 0,0780 & 0,2857 & 0,2722 \\
0 & 1 & 2 & 0,1066 & - & 0,0000 & - \\
0 & 1 & 1 & 0,1066 & - & 0,0000 & - \\
0 & 0 & 0 & 0,1066 & - & 0,0000 & - \\
\hline
\end{tabular}

Fuente: Elaboración propia a partir de la ECPF (1985-95).

Como resumen de las incidencias de las distintas características estudiadas en el proceso de salida de la pobreza, se observa que aquellos hogares con sustentador principal mujer permanecen más tiempo en pobreza que aquellos con sustentador principal hombre. Por otra parte, los hogares cuyo sustentador principal tiene nivel educativo superior ED4 (enseñanza superior), experimentan períodos más cortos de pobreza, mientras que aquellos con nivel educativo inferior ED1 (analfabetos y sin estudios) y ED2 (enseñanza primer ciclo) experimentan pobreza económica durante más tiempo. Del análisis es evidente que un hogar permanece menos tiempo en pobreza cuanto mayor sea el número de perceptores de ingresos. ${ }^{20}$ Para el resto de las variables, la interpretación es menos clara, dado que las curvas de supervivencia para cada una de las modalidades consideradas van intercambiando sus posiciones.

\section{CONCLUSIONES Y TRABAJOS FUTUROS}

En este trabajo, utilizando la Encuesta Continua de Presupuestos Familiares del período 1985-1995 en España, hemos realizado un análisis no paramétrico de la situación de la pobreza económica, así como un tratamiento estadístico de los datos que proporcionan dicha Encuesta. Destacamos la importancia de un análisis de corte transversal para cada trimestre, utilizando distintos índices de pobreza. No obstante, hemos considerado oportuno completar dicho análisis a través de la metodología propuesta por Jenkins y Lambert sobre las curvas TIP's, de gran utilidad para realizar comparaciones inter-temporales, como es nuestro caso. Queremos destacar, atendiendo a esta metodología, que los períodos expansivos que corres-

\footnotetext{
${ }^{20}$ Cantó (2001) obtiene conclusiones similares respecto a las variables que más inciden en la duración en el estado de pobreza. Los resultados obtenidos en el presente trabajo también ha sido observado en estudios de pobreza que utilizan distintas fuentes estadísticas [Bane and Ellwood (1986), Heinrich (1999)].
} 
pondieron a la economía española en el período 1985-1995 no fueron acompañados por una reducción en la intensidad de la pobreza.

Para el análisis longitudinal, hemos presentado un esquema metodológico basado en estimaciones de la función riesgo y la función de supervivencia, que permite una primera aproximación al análisis dinámico de la pobreza. Se observa que aproximadamente un $38 \%$ de hogares experimentaron pobreza económica más de un trimestre y que un $16 \%$ de los hogares muestrales se encontraron en estado de pobreza durante más de cuatro trimestres. Se ha observado también que la tasa de salida de la pobreza es decreciente, lo que refleja la existencia de dependencia de duración negativa a partir del primer trimestre, es decir, la probabilidad de salir de la pobreza disminuye a medida que aumentaba el tiempo en situación de pobreza.

No sólo cuántos, sino también quiénes son los pobres, constituyen preguntas obligadas en todo estudio de pobreza. Por este motivo nos ha interesado conocer cuáles son las características socioeconómicas y demográficas del hogar que pudieran afectar a la duración en el estado de pobreza en el período 1985-1995. Nos hemos preguntado si el "fenómeno de feminización" de la pobreza también se observa en aquellos hogares que experimentan situación de pobreza durante más de cuatro trimestres, pues las fuentes estadísticas de corte transversal reflejan una condición estática del "ser pobre" que tiende a enmascarar la fluidez y la progresión del proceso, por el que los hogares o individuos caen en pobreza no sólo una vez sino en varias ocasiones. Concluimos en el análisis que el fenómeno de "feminización de la pobreza" se mantiene en una perspectiva de panel, como quedó patente al contrastar la existencia de diferencias significativas entre las funciones de supervivencia según género. Además, observamos que aquellos hogares en los que el sustentador principal disfruta de un elevado potencial humano (experiencia laboral y nivel educativo) se enfrenta a períodos más cortos de pobreza que aquellos en los que el cabeza se encuentra "parado" o tiene "baja cualificación" y pertenece a la categoría de "analfabeto" y "sin estudios". La composición del hogar también importa en el proceso de salida de la pobreza. Como era de esperar aquellos hogares con mayor número de perceptores de ingresos experimentan períodos más cortos de pobreza. Así, y de acuerdo con los resultados obtenidos, el perfil de un hogar con duración en pobreza de más de cuatro trimestres, es un hogar con sustentador principal mujer, con un perceptor de ingreso que pertenece a la categoría laboral "Asalariado agrario sin especializar" o "Asalariado no agrario sin especializar", con nivel educativo "analfabeto", "sin estudios" o con enseñanza correspondiente al primer ciclo, y que habita en municipios de 50.001-500.000 habitantes.

Como trabajo futuro, pretendemos realizar un análisis paramétrico de las duraciones en pobreza en el período analizado que permita complementar el análisis no paramétrico realizado en este trabajo. En el nuevo enfoque se decidirá sobre la parametrización más adecuada para la variable duración y se incluirá además de las variables socioeconómicas y demográficas del hogar, variables estacionales dummies que permitan controlar las fluctuaciones a las que se ven sometidas los ingre- 
sos en los trimestres. Por otra parte, es interesante llevar a cabo un estudio similar para el decenio 1996-2005, de forma que nos permite contrastar los resultados obtenidos en ambos periodos y comprobar si el signo político de los gobiernos ha influido en el comportamiento de la pobreza.

\section{AGRADECIMIENTOS}

Este trabajo ha sido soportado en parte por el Ministerio de Ciencias y Tecnología por medio del proyecto TSI2006-13390-C02-02 y por la Junta de Andalucía por medio del proyecto de excelencia P06-TIC-02141 y la ayuda a consolidación de grupos 2006/SEJ-442.

\section{REFERENCIAS BIBLIOGRÁFICAS}

AHN, N. y UGIDOS-OLAZABAL, A. (1995): "Duration of unemployment in Spain: relative effects of unemployment benefits and family characteristics", Oxford Bulletin of Economics and Statistics, $\mathrm{n}^{\circ}$ 57, pp. 249-264.

BANE, M. y ELLWOOD, D. (1986): "Slipping into and out of poverty: The dynamics of spells", Journal of Human Resources, $n^{\circ} 21$ (1), pp. 1-23.

BÁRCENA MARTÍN, E. y COWELL, F.A. (2006): "Static and Dynamic Poverty in Spain, 19932000", Hacienda Pública Española, no 179-4, pp. 51-77.

BUHMANN, B.; RAINWATER, L.; SCHMAUS, G. y SMEEDING, T. (1988): "Equivalence scales, well-being, inequality and poverty", Review of Income and Wealth, $\mathrm{n}^{\circ} 34$, pp. $115-142$.

CANTÓ, O. (1996): "Poverty dynamics in Spain: A study of transitions in the 1990s", Technical Report, $n^{\circ} 15$, STICERD Analysis Research Programme.

CANTÓ, O. (2000): "Income mobility in Spain: How much is there?", Review of Income and Wealth , n $43(1)$, pp. 85-102.

CANTÓ, O. (2002): "Climbing out of poverty, falling back in: Low income stability in Spain", Applied Economics, $\mathrm{n}^{\circ} 34$ (15), pp. 1903-16.

CANTÓ, O.; DEL RÍO, C. y GRADÍN, C. (2003): "La Evolución de la pobreza estática y dinámica en España en el período 1985-1995", Hacienda Pública Española, n 167-4, pp. 87-119.

CES (1996): La pobreza y la exclusión social en España, Nices, Madrid.

CITRO, C. y MICHEL, R. (1995). Measuring Poverty: A new approach. National Academy Press, Washington D.C.

COX, D. (1972): "Regression models and life tables", Journal of the Royal Statistical Society, $\mathrm{n}^{\circ} 34$, Series B, pp. 187-220.

COX, D. Y OAKES, D. (1984): Analysis of Survival Data, Chapman and Hall, London.

DELEECK, K. y DE BOSCH, L. V. (1990): "Dimensión de la pobreza en un contexto comparativo: evidencia empírica y evaluación metodológica de cuatro umbrales de pobreza en siete países de la comunidad europea", Cuadernos de Acción Social, n² 24, pp. 76-107.

DEL RIO, C. y RUIZ-CASTILLO, J. (2001): "TIPs for Poverty Analysis. The case of Spain 1980-1981 to 1990-91", Investigaciones Económicas, vol. XXV (1), pp. 63-91.

DUNCAN, G.; GUSTAFSSON, B.; SCHMAUSS, G.; MESSINGER, H., H. MUFFELS y RAY, J. (1993): "Poverty dynamics in eight countries", Journal of Population Economics, $\mathrm{n}^{\circ}$ 6, pp. 215-234. 
ESCRIBANO, C. (1990): “Evolución de la pobreza en España 1973-1987”, Información Comercial Española, n 686, pp. 81-108.

FOSTER, J.; GREER, J. y THOBERCKE, E. (1984): "A class of decomposable poverty measures", Econometrica, $n^{\circ}$ 52, pp. 761-766.

GONZÁLEZ, M.R. y MARTÍN, D. (2006): "An econometric analysis of the characterization of poverty in Andalusia and in Spain: A comparative study", Scientific Bulletin of Chelm, $\mathrm{n}^{\circ} 2$, pp.161-177.

GONZÁLEZ, M.R.; MARTÍN, D.; GONZALEZ-ABRIL, L. y VELASCO F. (2007): "Un análisis transversal y longitudinal en el estado de pobreza en Alemania", Revista de Métodos Cuantitativos para la Economía y la Empresa, $n^{\circ} 4$, pp.7-34.

HECKAMAN, J. y SINGER, B. (1984): "Econometric duration analysis", Journal of Econometrics, $\mathrm{n}^{\circ} 24$ (1), pp. 63-132.

HEINRICH, G. (1999): "Does event history matter? a spell based approach to poverty dynamics in the United States and Germany", Econometric Society European Meeting, Santiago de Compostela.

HOSMER, D. y LEMESHOW, S. (1999): "Applied survival analysis: Regression modelling of time to event data", John Wiley Sons Inc., New York.

IMEDIO OLMEDO, L.J. y BARCENA MARTÍN, E. (2003): "Privación, status e imposición sobre la renta", Estudios de Economía Aplicada, n² 21-1; pp. 123-147.

INE (1985): Encuesta Continua de Presupuestos Familiares, INE, Madrid.

INE (1996): "Desigualdad y Pobreza en España”, Estudio Basado en las Encuestas de Presupuestos Familiares de 1973-74, 1980-81 y 1990-91, INE, Madrid.

INE (1997): Panel de Hogares de La Unión Europea, INE, Madrid.

JENKINS, S. (1999): "Modelling household income dynamics", Documento de Trabajo 99-9, ESRC Research Centre for Micro-Social Change.

JENKINS, S. (2000): "Modelling household dynamics", Journal of Population Economics, $\mathrm{n}^{\circ} 13$, pp. 529-567.

JENKINS, S. y LAMBERT, P. (1997): "Three i's of poverty curves with an analysis of UK poverty trends", Oxford Economic Papers, $\mathrm{n}^{\circ} 49$, pp. 317-327.

KALBFLEISCHE, J. y PRENTICE, R. (1980): The statistical analysis of failure time data, John Wiley y Sons Inc., New York.

KENNAN, J. (1985): "The duration of contract strikes in U.S. manufacturing", Journal of Econometrics, $\mathrm{n}^{\circ} 28$, pp. 5-28.

KIEFER, N. (1988): "Economic duration data y hazard functions", Journal of Economic Literature, $\mathrm{n}^{\circ} 26$, pp. 646-679.

LANCASTER, T. (1990): "The Econometric Analysis of Transition Data”, Cambridge University Pres, New York.

LAWLESS, J. (1982): Statistical models methods for lifetime data, John Wiley y Sons, New York.

LILLARD, L. y WILLIS, R. (1978): "Dynamic aspects of earning mobility", Econometrica, $n^{\circ} 46$, pp. 985-1012.

MERCADER, M. (1995): "The low income population in Spain and a comparison with France and UK. evidence from the household expenditures surveys", The Welfare State Programme, wsp/95.

NEWMAN, J. y CULLOUGH, C. M. (1984): "A hazard rate approach to the timing of births", Econometrica, $\mathrm{n}^{\circ}$ 52, pp. 939-962.

NICKELL, S. y LANCASTER, T. (1980): "The analysis of re-employment probabilities of the unemployed", Journal of the Royal Statistical Society 52 Series A, vol. 143, $\mathrm{n}^{\circ} 2$, pp. 141-165.

PAKES, A. y SIMPSON, M. (1989): Patent renewal data, Technical Report, Brooking Papers on Economic Activity, Microeconomics.

PRENTICE, R. y GLOEKLER, L. (1974): "Regression analysis of grouped survival data with application to breast cancer data", Biometrics, $\mathrm{n}^{\circ} 34$, pp.57-67. 
RODGERS, J. y RODGERS, J. (1993): "Chronic poverty in the U.S.", Journal of Human Resources, $\mathrm{n}^{\circ} 28, \mathrm{pp} .25-54$.

RUIZ-HUERTA, J. y MARTÍNEZ, R. (1994): "La Pobreza en España: ¿Qué nos muestran las Encuestas de Presupuestos Familiares?", Documentación Social, nº 96, pp. 15-111.

RUGGLES, P. y ROBERSTON, W. (1989): "Longitudinal measures of poverty: Accounting for income and assets over time", Review of Income and Wealth, $\mathrm{n}^{\circ} 3$ Series 35, pp.225-264.

SEN, A. (1976): "Poverty: An ordinal approach to measurement", Econometrica, $\mathrm{n}^{\circ} 44$ (2), pp. 219-231.

SEN, A. (1978): "Three notes on the concept of poverty", Technical Report, $n^{\circ} 65$, International Labour Office, Geneva.

SEN, A. (1979): "Issues in the measurement of poverty", Scandinavian Journal of Economics, $\mathrm{n}^{\circ} 81$, pp. 285-307.

STEVENS, A. (1994): "The dynamics of poverty spells: Updating Bane and Ellwood",American Economic Review, $\mathrm{n}^{\circ} 84$ (2), pp. 34-37.

STEVENS, A. (1999): "Climbing out of poverty, falling back", Journal of Human Resources, $\mathrm{n}^{\circ} 34$, pp. 557-588.

WALKER, R. (1994): "Poverty Dynamics: Issues and Examples", Avebury, Aldershot, England. 